\title{
Modeling dimensionally-heterogeneous problems: analysis, approximation and applications
}

\author{
Pablo J. Blanco • Marco Discacciati • \\ Alfio Quarteroni
}

\begin{abstract}
In the present work a general theoretical framework for coupled dimensionally-heterogeneous partial differential equations is developed. This is done by recasting the variational formulation in terms of coupling interface variables. In such a general setting we analyze existence and uniqueness of solutions for both the continuous problem and its finite dimensional approximation. This approach also allows the development of different iterative substructuring solution methodologies involving
\end{abstract}

The first author acknowledges the support of the Brazilian agencies CNPq and FAPERJ. The second and third authors acknowledge the European Research Council Advanced Grant "Mathcard, Mathematical Modelling and Simulation of the Cardiovascular System”, Project ERC-2008-AdG 227058.

P. J. Blanco

LNCC, Laboratório Nacional de Computação Científica, Av. Getúlio Vargas 333, Quitandinha, 25651-075 Petrópolis, Brazil

e-mail: pjblanco@lncc.br

M. Discacciati $(\varangle) \cdot$ A. Quarteroni

MATHICSE, Chair of Modelling and Scientific Computing, Ecole Polytechnique Fédérale de Lausanne, Station 8, CH-1015 Lausanne, Switzerland e-mail: marco.discacciati@epfl.ch
A. Quarteroni
e-mail: alfio.quarteroni@epfl.ch

Present Address:

M. Discacciati

Laboratori de Càlcul Numèric, Universitat Politècnica de Catalunya,

C/Jordi Girona 1-3, Edifici C2, 08034 Barcelona, Spain

e-mail: marco.discacciati@upc.edu

A. Quarteroni

MOX, Politecnico di Milano, P.zza Leonardo da Vinci 32, 20133 Milano, Italy 
dimensionally-homogeneous subproblems. Numerical experiments are carried out to test our theoretical results.

Mathematics Subject Classification (2000) $\quad 35 \mathrm{~J} 20 \cdot 65 \mathrm{~N} 55 \cdot 65 \mathrm{~N} 30$

\section{Introduction}

The geometrical multiscale modeling, that is the use of dimensionally-heterogeneous representations of different physical systems, has been successfully applied in the past few years in different fields [5-8, 12,14,16,19,24]. The appealing aspect of such an approach is that it allows for the interaction between different geometrical scales in a given system. For instance, in the context of the cardiovascular system this allows for the integrated modeling of the hemodynamics, taking into account the interplay between the global systemic dynamics and the complex local blood flow behavior $[5,7,8,12,14,24]$.

Although domain decomposition methods are commonplace in practice when coupling dimensionally homogeneous models, dimensionally heterogeneous models have made the object of a rigorous analysis only sporadically (see for example the recent publication [15]).

Motivated by the relevance of such models in several applications, and because of the lack of a general analysis, in the present we aim at: (1) providing a general framework for such kind of problems as well as to carry out an abstract analysis including a study of existence and uniqueness of solutions in the continuous and in the discrete cases, and (2) carrying out a systematic construction of partitioning methodologies in the context of domain decomposition methods. As a matter of fact, some alternative possibilities to those encountered in the classical domain decomposition literature, specifically devised for the dimensionally-heterogeneous case, are presented and discussed. Regarding this last point we will set the baseline on top of which the partitioning methodologies which are proposed in [15] are built.

In order to see where we stand for with the analysis and examples presented in this work, in Fig. 1 we summarize the different contexts in which domain decompo-

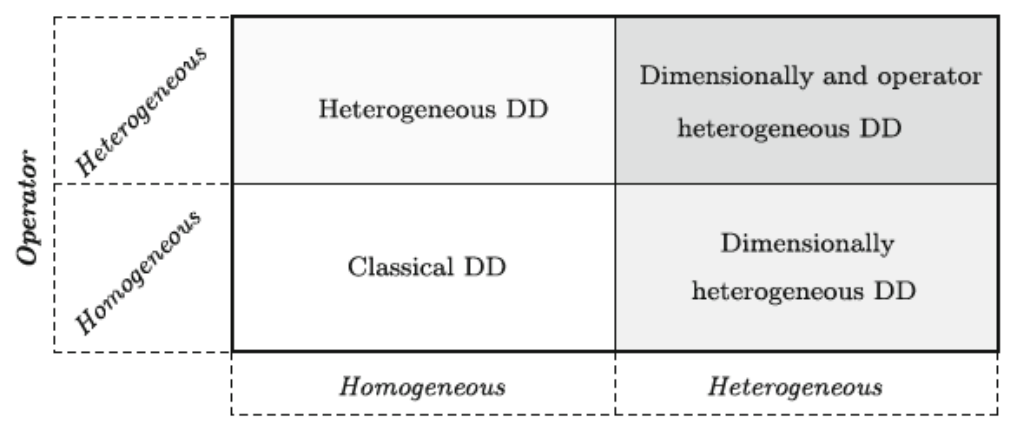

Geometric dimension

Fig. 1 Application of domain decomposition concepts to different modeling problems 
sition strategies can be employed. Particularly, we point out that the construction of a model comprises the definition of two basic elements which determine its nature: (1) the differential operator which represents the main physical phenomenon, and (2) the dimension of the Euclidean space in which such operator is going to be considered. Classical domain decomposition methods were born in the setting of models sharing the same operator in the same Euclidean space (see [18,22,23] and references therein). Heterogeneous domain decomposition methods (see [18, Chapter 8] for some examples) are referred to those cases in which the differential operators are not the same in different regions of the computational domain. In this category we can include also the fluid-structure interaction coupling, Stokes-Darcy coupling, pure advection and advection-diffusion coupling, among others (see, e.g., $[2,9,10,13,20]$ ). On the other hand, when models with different geometrical dimensions are employed, this is referred to as a dimensionally-heterogeneous domain decomposition method.

In this paper, we will show the way the coupling of elliptic dimensionallyheterogeneous operators fits within such an abstract setting. Concerning the applications we present numerical examples of a 2D-1D coupled problem involving also the Laplace operators and a 3D-1D coupled problem in the field of linear elasticity. These examples are employed to test the validity of our theoretical results.

The present work is organized as follows. In Sect. 2 we formulate the general problem. Section 3 presents, for the simplest configuration of two coupled heterogeneous models, some theoretical results about existence and uniqueness and also provides the guidelines for setting up partitioning methods for the segregated solution of these problems. In Sect. 4 we extend the framework and the corresponding results for some cases involving multi-component systems, while in Sect. 5 the discrete problem is addressed and some results are developed. Numerical experiments rendering some applications and testing the theoretical results are elaborated in Sect. 6. Finally, the main conclusions of the work are drawn in Sect. 7.

\section{Abstract setting for heterogeneous coupling}

\subsection{Preliminaries}

Let us assume that a physical system is split into two parts and that, based on the characteristics of the system itself, one of the two parts can be described via a dimensionally reduced model. A three-dimensional hydraulic network is a clear example where some of the pipes can be described by simplified 0D algebraic relations between flow and pressure drop, or by any other simple representation instead of considering, e.g., the full Navier-Stokes equations in 3D. In abstract terms we deal with two kinds of models that will be referred to as complex dimensional and simple dimensional models, or in compact form, $\mathbb{C D}$-model and $\mathbb{S D}$-model. Generally speaking we can consider a wide range of combinations of the form $\mathbb{C D}-\mathbb{S D}$ with $\mathbb{C}=1,2,3$ and $\mathbb{S}=0,1,2$. In this context we will speak of admissible combination when $\mathbb{C}>\mathbb{S}$. Therefore, we can have situations like the coupling of 3D-2D models, where in this case the $2 \mathrm{D}$ acts as the simple model, or 1D-0D models where the $1 \mathrm{D}$ is the complex representation.

From now on we will stick to the following assumptions for the sake of boundedness in the work. 


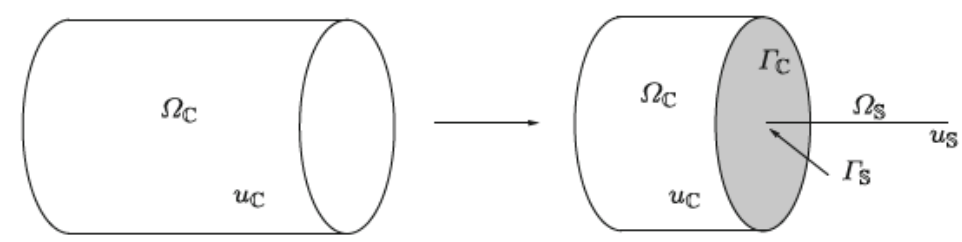

Fig. 2 Scheme of the geometrical and mathematical setting

Assumption 1 We consider the cases $\mathbb{C}=1,2,3$ and $\mathbb{S}=0,1,2$ satisfying $\mathbb{C}>\mathbb{S}$ (admissible combinations).

Assumption 2 We consider only two models at the same time in a given system, that is one $\mathbb{C D}$-model and one $\mathbb{S D}$-model. More general situations could involve, for instance 3D-1D-0D representations for different parts of the system. There is no loss of generality due to this last assumption.

In the first part of this work we develop all the theoretical results for a representation involving two dimensionally-heterogeneous models, that is a system with one single coupling interface. The extension to multi-component systems is carried out at a later stage.

\subsection{Extended variational formulation for heterogeneous coupling}

Let $\mathcal{U}_{\mathbb{C}}$ be a Hilbert space on the domain $\Omega_{\mathbb{C}}, \hat{U}_{\mathbb{C}} \subseteq \mathcal{U}_{\mathbb{C}}$ be a subspace of $\mathcal{U}_{\mathbb{C}}$ and $U_{\mathbb{C}} \subseteq \mathcal{U}_{\mathbb{C}}$ be the affine manifold associated to $\hat{U}_{\mathbb{C}}$ which accounts for the boundary conditions of the problem of interest.

Let us consider the following dimensionally-homogeneous variational problem corresponding to the $\mathbb{C D}$-model defined in a domain $\Omega_{\mathbb{C}}$ of the Euclidean space $\mathbb{R}^{d}(d=$ 1, 2,3): find $u_{\mathbb{C}} \in U_{\mathbb{C}}$ such that

$$
a_{\mathbb{C}}\left(u_{\mathbb{C}}, \hat{u}_{\mathbb{C}}\right)=f_{\mathbb{C}}\left(\hat{u}_{\mathbb{C}}\right) \quad \forall \hat{u}_{\mathbb{C}} \in \hat{U}_{\mathbb{C}}
$$

where $a_{\mathbb{C}}: \mathcal{U}_{\mathbb{C}} \times \mathcal{U}_{\mathbb{C}} \rightarrow \mathbb{R}$ is a bilinear continuous form, and $f_{\mathbb{C}}: \mathcal{U}_{\mathbb{C}} \rightarrow \mathbb{R}$ is a linear continuous functional. We assume that $a_{\mathbb{C}}$ is coercive on $\hat{U}_{\mathbb{C}}$ with respect to the norm $\|\cdot\| \mathcal{u}_{\mathbb{C}}$. In the rest of the paper we will denote by $\|v\|_{\hat{U}_{\mathbb{C}}}$ and $\|w\|_{U_{\mathbb{C}}}$ the norm of $v \in \hat{U}_{\mathbb{C}}$ and $w \in U_{\mathbb{C}}$, respectively. Notice that these norms are equivalent to the norm $\|\cdot\|_{\mathcal{U}_{\mathbb{C}}}$.

Assume now that one part of the domain $\Omega_{\mathbb{C}}$ is replaced by a S-dimensional domain $\Omega_{\mathbb{S}}$ where, instead of $u_{\mathbb{C}}$, we have the unknown $u_{\mathbb{S}} \in U_{\mathbb{S}}$. The $\mathbb{C D}$ and the SD models are suitably coupled through the coupling interfaces, $\Gamma_{\mathbb{C}}$ and $\Gamma_{\mathbb{S}}$, as made clear later.

A schematic figure of the modeling problem we are addressing here is shown in Fig. 2.

We need to identify the trace spaces over such interfaces denoted by $\Lambda_{\mathbb{C}}$ and $\Lambda_{\mathbb{S}}$ and the corresponding dual spaces $\Lambda_{\mathbb{C}}^{\prime}$ and $\Lambda_{\mathbb{S}}^{\prime}$.

Moreover, we consider the following restriction operator

$$
\mathcal{R}_{\mathbb{S}}: \Lambda_{\mathbb{C}} \rightarrow \Lambda_{\mathbb{S}}, \quad u_{\mathbb{C} \mid \Gamma_{\mathbb{C}}} \mapsto \mathcal{R}_{\mathbb{S}} u_{\mathbb{C} \mid \Gamma_{\mathbb{C}}}
$$


This operator must be surjective, but not necessarily injective, so in general it is not invertible. Indeed, we may have $u_{\mathbb{C}}^{1}, u_{\mathbb{C}}^{2} \in U_{\mathbb{C}}, u_{\mathbb{C}}^{1} \neq u_{\mathbb{C}}^{2}$, such that $\mathcal{R}_{\mathbb{S}} u_{\mathbb{C} \mid \Gamma_{\mathbb{C}}}^{1}=$ $\mathcal{R}_{\mathbb{S}} u_{\mathbb{C} \mid \Gamma_{\mathbb{C}}}^{2}$

Furthermore, we introduce the following extension operator

$$
\mathcal{E}_{\mathbb{C}}: \Lambda_{\mathbb{S}} \rightarrow \Lambda_{\mathbb{C}}, \quad u_{\mathbb{S} \mid \Gamma_{\mathbb{S}}} \mapsto \mathcal{E}_{\mathbb{C}} u_{\mathbb{S} \mid \Gamma_{\mathbb{S}}}
$$

In turn, this operator must be injective, but in general not necessarily surjective, therefore, it is not invertible. Both the restriction and extension operators are linear and continuous.

From now on we will omit the notations $\mid \Gamma_{\mathbb{C}}$ and $\mid \Gamma_{\mathbb{S}}$ since it will always be clear from the context on which interface we are working.

Let $\mathcal{U}_{\mathbb{S}}$ be a Hilbert space on the domain $\Omega_{\mathbb{S}}, \hat{U}_{\mathbb{S}} \subseteq \mathcal{U}_{\mathbb{S}}$ be a subspace of $\mathcal{U}_{\mathbb{S}}$ and $U_{\mathbb{S}} \subseteq \mathcal{U}_{\mathbb{S}}$ be the affine manifold associated to $\hat{U}_{\mathbb{S}}$ which accounts for the boundary conditions.

The variational problem for the coupled dimensionally-heterogeneous model reads: for a given $\alpha \in\{0,1\}$ a priori defined, find $\left(u_{\mathbb{C}}, u_{\mathbb{S}}\right) \in U_{\mathbb{C}, \mathbb{S}}$ such that

$$
a_{\mathbb{C}}\left(u_{\mathbb{C}}, \hat{u}_{\mathbb{C}}\right)+a_{\mathbb{S}}\left(u_{\mathbb{S}}, \hat{u}_{\mathbb{S}}\right)=f_{\mathbb{C}}\left(\hat{u}_{\mathbb{C}}\right)+f_{\mathbb{S}}\left(\hat{u}_{\mathbb{S}}\right) \quad \forall\left(\hat{u}_{\mathbb{C}}, \hat{u}_{\mathbb{S}}\right) \in \hat{U}_{\mathbb{C}, \mathbb{S}}
$$

where the linear space $\hat{U}_{\mathbb{C}, \mathbb{S}}$ is defined by

$$
\begin{gathered}
\hat{U}_{\mathbb{C}, \mathbb{S}}=\left\{\left(\hat{u}_{\mathbb{C}}, \hat{u}_{\mathbb{S}}\right) \in \hat{U}_{\mathbb{C}} \times \hat{U}_{\mathbb{S}}: \alpha\left(\hat{u}_{\mathbb{S}}-\mathcal{R}_{\mathbb{S}} \hat{u}_{\mathbb{C}}\right)=0 \text { on } \Gamma_{\mathbb{S}}\right. \\
\left.(1-\alpha)\left(\hat{u}_{\mathbb{C}}-\mathcal{E}_{\mathbb{C}} \hat{u}_{\mathbb{S}}\right)=0 \text { on } \Gamma_{\mathbb{C}}\right\}
\end{gathered}
$$

In (1) we have that $a_{\mathbb{S}}: \mathcal{U}_{\mathbb{S}} \times \mathcal{U}_{\mathbb{S}} \rightarrow \mathbb{R}$ is a bilinear, continuous form, coercive on $\hat{U}_{\mathbb{S}}$ with respect to the norm $\|\cdot\|_{\mathcal{U}_{\mathbb{S}}}$, while $f_{\mathbb{S}}: \mathcal{U}_{\mathbb{S}} \rightarrow \mathbb{R}$ is a linear and continuous functional. Note that there are two constraints in the linear space $\hat{U}_{\mathbb{C}, \mathbb{S}}$ which account for the continuity of the traces in two different senses given by the trace spaces $\Lambda_{\mathbb{C}}$ and $\Lambda_{\mathbb{S}}$. Nevertheless, it is actually just one constraint at once that is active since $\alpha$ is either 0 or 1 .

Let us reformulate problem (1) by relaxing both restrictions $\hat{u}_{\mathbb{S}}=\mathcal{R}_{\mathbb{S}} \hat{u}_{\mathbb{C}}$ on $\Gamma_{\mathbb{S}}$ and $\hat{u}_{\mathbb{C}}=\mathcal{E}_{\mathbb{C}} \hat{u}_{\mathbb{S}}$ on $\Gamma_{\mathbb{C}}$ through dual variables that act as Lagrange multipliers.

More precisely, we formulate the augmented variational formulation as follows: for a given $\alpha \in\{0,1\}$ a priori defined, find $\left(u_{\mathbb{C}}, u_{\mathbb{S}}, \lambda_{\mathbb{C}}, \lambda_{\mathbb{S}}\right) \in U_{\mathbb{C}} \times U_{\mathbb{S}} \times \Lambda_{\mathbb{C}}^{\prime} \times \Lambda_{\mathbb{S}}^{\prime}$ such that

$$
\begin{aligned}
& a_{\mathbb{C}}\left(u_{\mathbb{C}}, \hat{u}_{\mathbb{C}}\right)+a_{\mathbb{S}}\left(u_{\mathbb{S}}, \hat{u}_{\mathbb{S}}\right) \\
& \quad+(1-\alpha)\left\langle\lambda_{\mathbb{C}}, \hat{u}_{\mathbb{C}}-\mathcal{E}_{\mathbb{C}} \hat{u}_{\mathbb{S}}\right\rangle_{\mathbb{C}}+(1-\alpha)\left\langle\hat{\lambda}_{\mathbb{C}}, u_{\mathbb{C}}-\mathcal{E}_{\mathbb{C}} u_{\mathbb{S}}\right\rangle_{\mathbb{C}} \\
& \quad+\alpha\left\langle\lambda_{\mathbb{S}}, \hat{u}_{\mathbb{S}}-\mathcal{R}_{\mathbb{S}} \hat{u}_{\mathbb{C}}\right\rangle_{\mathbb{S}}+\alpha\left\langle\hat{\lambda}_{\mathbb{S}}, u_{\mathbb{S}}-\mathcal{R}_{\mathbb{S}} u_{\mathbb{C}}\right\rangle_{\mathbb{S}} \\
& \quad=f_{\mathbb{C}}\left(\hat{u}_{\mathbb{C}}\right)+f_{\mathbb{S}}\left(\hat{u}_{\mathbb{S}}\right) \quad \forall\left(\hat{u}_{\mathbb{C}}, \hat{u}_{\mathbb{S}}, \hat{\lambda}_{\mathbb{C}}, \hat{\lambda}_{\mathbb{S}}\right) \in \hat{U}_{\mathbb{C}} \times \hat{U}_{\mathbb{S}} \times \Lambda_{\mathbb{C}}^{\prime} \times \Lambda_{\mathbb{S}}^{\prime},
\end{aligned}
$$


where the symbols $\langle\cdot, \cdot\rangle_{\mathbb{C}}$ and $\langle\cdot, \cdot\rangle_{\mathbb{S}}$ denote the duality pairings:

$$
\langle\cdot, \cdot\rangle_{\mathbb{C}}: \Lambda_{\mathbb{C}}^{\prime} \times \Lambda_{\mathbb{C}} \rightarrow \mathbb{R} \text { and }\langle\cdot, \cdot\rangle_{\mathbb{S}}: \Lambda_{\mathbb{S}}^{\prime} \times \Lambda_{\mathbb{S}} \rightarrow \mathbb{R}
$$

Remark 1 An alternative approach would be to work in the spaces $\Lambda_{\mathbb{C}}$ and $\Lambda_{\mathbb{S}}$ for both unknowns and test functions and to replace dualities $\langle\cdot, \cdot\rangle_{\mathbb{S}}$ and $\langle\cdot, \cdot\rangle_{\mathbb{C}}$ by scalar products $(\cdot, \cdot)_{\mathbb{C}}$ and $(\cdot, \cdot)_{\mathbb{S}}$ by making use of the Riesz representation theorem. In such case, all proofs given in the forthcoming sections can be consistently modified in order to get the corresponding results.

Finally, we introduce the adjoint operators $\mathcal{R}_{\mathbb{S}}^{*}$ and $\mathcal{E}_{\mathbb{C}}^{*}$ of $\mathcal{R}_{\mathbb{S}}$ and $\mathcal{E}_{\mathbb{C}}$, respectively, such that there hold

$$
\begin{aligned}
& \left\langle\lambda_{\mathbb{C}}, \mathcal{E}_{\mathbb{C}} u_{\mathbb{S}}\right\rangle_{\mathbb{C}}=\left\langle\mathcal{E}_{\mathbb{C}}^{*} \lambda_{\mathbb{C}}, u_{\mathbb{S}}\right\rangle_{\mathbb{S}} \quad \forall\left(u_{\mathbb{S}}, \lambda_{\mathbb{C}}\right) \in \Lambda_{\mathbb{S}} \times \Lambda_{\mathbb{C}}^{\prime} \\
& \left\langle\lambda_{\mathbb{S}}, \mathcal{R}_{\mathbb{S}} u_{\mathbb{C}}\right\rangle_{\mathbb{S}}=\left\langle\mathcal{R}_{\mathbb{S}}^{*} \lambda_{\mathbb{S}}, u_{\mathbb{C}}\right\rangle_{\mathbb{C}} \quad \forall\left(u_{\mathbb{C}}, \lambda_{\mathbb{S}}\right) \in \Lambda_{\mathbb{C}} \times \Lambda_{\mathbb{S}}^{\prime} .
\end{aligned}
$$

The characterization of these operators together with that of $\mathcal{R}_{\mathbb{S}}$ and $\mathcal{E}_{\mathbb{C}}$, in each specific problem, is fundamental to set up the domain decomposition framework and, in particular, to define the extension operators of Sects. 3.2 and 3.3.

At this point we can establish an analogy with similar concepts from solid mechanics, where the dimensional reduction of the model has a direct connection to constraints introduced in the definition of the kinematics of the structure. In this sense, the dimensional heterogeneity of the structure can be understood as the result of the coexistence of different kinematics assumptions which must be matched at the coupling interfaces through suitable coupling conditions (see [6] for a perspective in the field of solid mechanics). For instance, if we couple a 3D solid model and a shell model under some hypotheses, say Kirchhoff-Love hypotheses, we are trying to match a fully 3D kinematics and a constrained kinematics consisting of tangent and normal displacements and tangent rotations (tangent and normal refer to the mid surface of the shell), which leads to a $2 \mathrm{D}$ theory of solid mechanics. Thus, in such case we have a heterogeneous model embodying two different kinematics.

\subsection{Example of application 1: coupling 3D-1D}

Let us consider a 1D Laplace problem set up in a 1D domain $\Xi$ (corresponding to $\Omega_{\mathbb{S}}$ ) coupled with a 3D Laplace problem set up in a 3D domain $\Omega$ (corresponding to $\Omega_{\mathbb{C}}$ ) (like, e.g., in Fig. 2, right). This can be a simple paradigm to describe a steady diffusion process in a structure represented by heterogeneous 3D and 1D models. The coupling interface is characterized by two elements. From the 3D domains the interface $\Gamma_{\mathbb{C}}$ is a surface here denoted by $\Gamma$, while from the 1D counterpart $\Gamma_{\mathbb{S}}$ is a point denoted by $\gamma$. Moreover, we have $U_{\mathbb{C}}=\left\{v \in H^{1}(\Omega): v=0\right.$ on $\left.\partial \Omega \backslash \Gamma\right\}, \Lambda_{\mathbb{C}}=H^{1 / 2}(\Gamma), \Lambda_{\mathbb{C}}^{\prime}=$ $H^{-1 / 2}(\Gamma), U_{\mathbb{S}}=H^{1}(\Xi)+$ b.c., $\Lambda_{\mathbb{S}}=\mathbb{R}$, and $\Lambda_{\mathbb{S}}^{\prime}=\mathbb{R}$. The fields are denoted by $u_{\mathbb{C}}=u_{3}$ and $u_{\mathbb{S}}=u_{1}$ referring to the $3 \mathrm{D}$ and $1 \mathrm{D}$ solutions respectively. The bilinear 
and linear forms are then defined as:

$$
\begin{array}{ll}
a_{\mathbb{C}}\left(u_{\mathbb{C}}, \hat{u}_{\mathbb{C}}\right)=\int_{\Omega} k \nabla u_{3} \cdot \nabla \hat{u}_{3} \mathrm{~d} \Omega, \quad f_{\mathbb{C}}\left(\hat{u}_{\mathbb{C}}\right)=\int_{\Omega} f \hat{u}_{3} \mathrm{~d} \Omega, \\
a_{\mathbb{S}}\left(u_{\mathbb{S}}, \hat{u}_{\mathbb{S}}\right)=\int_{\Xi} A k \frac{d u_{1}}{d \xi} \frac{d \hat{u}_{1}}{d \xi} \mathrm{d} \Xi, \quad f_{\mathbb{S}}\left(\hat{u}_{\mathbb{S}}\right)=\int_{\Xi} A f \hat{u}_{1} \mathrm{~d} \Xi,
\end{array}
$$

where $A$ is a scaling factor in the $\mathbb{S D}$-model corresponding to the cross-sectional area of the $\mathbb{C D}$-model through which the reduction has been performed. Here we considered the material property $k$ and the source term $f$ constants in both the 3D and the 1D regions. In addition, the operator $\mathcal{R}_{\mathbb{S}}$ may be defined in the following manner

$$
\mathcal{R}_{\mathbb{S}}: H^{1 / 2}(\Gamma) \rightarrow \mathbb{R}, \quad u_{3 \mid \Gamma} \mapsto u_{3,1 \mid \gamma}=\frac{1}{|\Gamma|} \int_{\Gamma} u_{3} \mathrm{~d} \Gamma
$$

which is clearly a surjective operator, whereas the operator $\mathcal{E}_{\mathbb{C}}$ may be given by

$$
\mathcal{E}_{\mathbb{C}}: \mathbb{R}: \rightarrow H^{1 / 2}(\Gamma), \quad u_{1 \mid \gamma} \mapsto u_{1,3 \mid \Gamma}=u_{1 \mid \gamma},
$$

being this an injective operator. Note that $u_{1,3}$ is a constant function defined in all $\Gamma$. Finally, the duality pairings in this case are

$$
\begin{aligned}
\left\langle\lambda_{\mathbb{C}}, \hat{u}_{\mathbb{C}}-\mathcal{E}_{\mathbb{C}} \hat{u}_{\mathbb{S}}\right\rangle_{\mathbb{C}}={ }_{\left(H^{1 / 2}(\Gamma)\right)^{\prime}\left\langle\lambda_{3}, \hat{u}_{3}-\hat{u}_{1,3}\right\rangle_{H^{1 / 2}(\Gamma)},}, \\
\left\langle\lambda_{\mathbb{S}}, \hat{u}_{\mathbb{S}}-\mathcal{R}_{\mathbb{S}} \hat{u}_{\mathbb{C}}\right\rangle_{\mathbb{S}}=|\Gamma| \lambda_{1}\left(\hat{u}_{1}-\hat{u}_{3,1}\right)_{\mid \gamma},
\end{aligned}
$$

where the factor $|\Gamma|$ is included so that both Lagrange multipliers have the same physical dimension. In this case $\lambda_{1} \in \mathbb{R}$.

For this problem, the differential equations are the following:

$$
\begin{cases}-\operatorname{div}\left(k \nabla u_{3}\right)=f & \text { in } \Omega, \\ -\frac{d}{d \xi}\left(A k \frac{d u_{1}}{d \xi}\right)=A f & \text { in } \Xi, \\ \text { 3D boundary conditions } & \text { in } \partial \Omega \backslash \Gamma, \\ 1 \mathrm{D} \text { boundary conditions } & \text { in } \partial \backslash \gamma,\end{cases}
$$

whereas the coupling conditions are

$$
\begin{aligned}
& \text { if } \alpha=1 \begin{cases}u_{1}=\frac{1}{|\Gamma|} \int_{\Gamma} u_{3} \mathrm{~d} \Gamma & \text { in } \gamma, \\
k \frac{d u_{1}}{d \xi}=k \nabla u_{3} \cdot \mathbf{n} & \text { on } \Gamma,\end{cases} \\
& \text { if } \alpha=0 \quad \begin{cases}u_{1}=u_{3} \\
A k \frac{d u_{1}}{d \xi}=\int_{\Gamma} k \nabla u_{3} \cdot \mathbf{n} \mathrm{d} \Gamma & \text { in } \gamma .\end{cases}
\end{aligned}
$$

The equalities on $\Gamma$ here above must be intended in the sense of traces. 


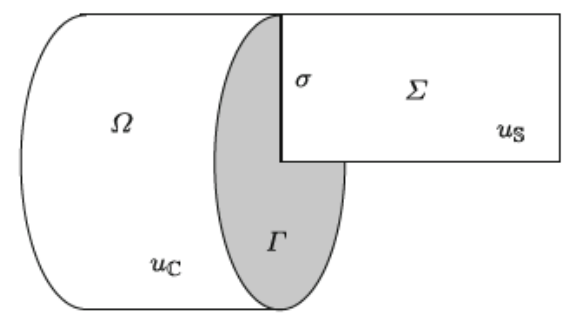

Fig. 3 Setting of the 3D-2D coupled problem

In the 3D-1D example just presented, the interface variables of the SD-model belong to the finite-dimensional space $\mathbb{R}$. This will lead to some special behavior in the discrete case as we will see in forthcoming sections. Unlike this, we are going to present a $3 \mathrm{D}-2 \mathrm{D}$ example in the next section where the interface variables remain in an infinitedimensional space.

\subsection{Example of application 2: Coupling 3D-2D}

Let us formulate now the coupling between a 2D axisymmetric Laplace problem set up in a $2 \mathrm{D}$ domain, for which $\Omega_{\mathbb{S}}$ is a $2 \mathrm{D}$ domain denoted by $\Sigma((r, z)$ are the radial and axial coordinates respectively), with a $3 D$ Laplace problem, for which $\Omega_{\mathbb{C}}$ is a $3 D$ domain denoted by $\Omega$. Here the coupling interface $\Gamma_{\mathbb{C}}$ is a surface denoted by $\Gamma$ while $\Gamma_{\mathbb{S}}$ is a straight line denoted by $\sigma$ (see Fig. 3).

In this case we have $U_{\mathbb{C}}=\left\{v \in H^{1}(\Omega): v=0\right.$ on $\left.\partial \Omega \backslash \Gamma\right\}, \Lambda_{\mathbb{C}}=H_{00}^{1 / 2}(\Gamma)$, $\Lambda_{\mathbb{C}}^{\prime}=H^{-1 / 2}(\Gamma), U_{\mathbb{S}}=\left\{v \in H_{r}^{1}(\Sigma): v=0\right.$ on $\left.\partial \Sigma \backslash \sigma\right\}$, where the weighted space $H_{r}^{1}(\Sigma)$ (see [3]) is the set of measurable functions $v$ with the norm

$$
\|v\|_{H_{r}^{1}(\Sigma)}^{2}=\sum_{\ell=0}^{1} \sum_{k=0}^{\ell}\left\|\partial_{r}^{k} \partial_{z}^{\ell-k} v\right\|_{L_{r}^{2}(\Sigma)}^{2} \text { and }\|v\|_{L_{r}^{2}(\Sigma)}^{2}=\int_{\Sigma} v^{2}(r, z) r \mathrm{~d} r \mathrm{~d} z .
$$

The associated trace space is $\Lambda_{\mathbb{S}}=\left\{v_{\mid \sigma}: v \in H_{r}^{1}(\Sigma)\right\}$ and $\Lambda_{\mathbb{S}}$ is its dual space. The unknown fields are now denoted by $u_{\mathbb{C}}=u_{3}$ and $u_{\mathbb{S}}=u_{2}$ referring to the $3 \mathrm{D}$ and $2 \mathrm{D}$ solutions respectively. Therefore, the bilinear and linear forms become:

$$
\begin{aligned}
a_{\mathbb{C}}\left(u_{\mathbb{C}}, \hat{u}_{\mathbb{C}}\right) & =\int_{\Omega} k \nabla u_{3} \cdot \nabla \hat{u}_{3} \mathrm{~d} \Omega, \\
a_{\mathbb{S}}\left(u_{\mathbb{S}}, \hat{u}_{\mathbb{S}}\right) & =\int_{\Sigma} 2 \pi r k\left[\frac{\partial u_{2}}{\partial r} \frac{\partial \hat{u}_{2}}{\partial r}+\frac{\partial u_{2}}{\partial z} \frac{\partial \hat{u}_{2}}{\partial z}\right] \mathrm{d} r \mathrm{~d} z, \\
f_{\mathbb{C}}\left(\hat{u}_{\mathbb{C}}\right) & =\int_{\Omega} f \hat{u}_{3} \mathrm{~d} \Omega,
\end{aligned}
$$




$$
f_{\mathbb{S}}\left(\hat{u}_{\mathbb{S}}\right)=\int_{\Sigma} 2 \pi r f \hat{u}_{2} \mathrm{~d} r \mathrm{~d} z
$$

where the scaling factor $2 \pi r$ accounts for the reduced representation with respect to the circumferential coordinate that is taken into account in the 2D-model. The material property $k$ and the source term $f$ are both constant in the 3D and 2D regions. In the present situation, the operator $\mathcal{R}_{\mathbb{S}}$ may simply be the average operator defined as follows

$$
\mathcal{R}_{\mathbb{S}}: H^{1 / 2}(\Gamma) \rightarrow H_{r}^{1 / 2}(\sigma), \quad u_{3 \mid \Gamma} \mapsto u_{3,2 \mid \sigma}=\frac{1}{2 \pi} \int_{0}^{2 \pi} u_{3} \mathrm{~d} \phi
$$

while the extension operator $\mathcal{E}_{\mathbb{C}}$ may be given by

$$
\mathcal{E}_{\mathbb{C}}: H_{r}^{1 / 2}(\sigma) \rightarrow H^{1 / 2}(\Gamma), \quad u_{2 \mid \sigma} \mapsto u_{2,3 \mid \Gamma}=u_{2 \mid \sigma}
$$

Notice that in this case $u_{2,3}$ is a function defined in all $\Gamma$ which varies with the radial coordinate but it is constant with respect to the circumferential coordinate. Finally, in this case the duality pairings read

$$
\begin{aligned}
& \left\langle\lambda_{\mathbb{C}}, \hat{u}_{\mathbb{C}}-\mathcal{E}_{\mathbb{C}} \hat{u}_{\mathbb{S}}\right\rangle_{\mathbb{C}}={ }_{H^{-1 / 2}(\Gamma)}\left\langle\lambda_{3}, \hat{u}_{3}-\hat{u}_{2,3}\right\rangle_{H_{00}}{ }^{1 / 2}(\Gamma) \\
& \left\langle\lambda_{\mathbb{S}}, \hat{u}_{\mathbb{S}}-\mathcal{R}_{\mathbb{S}} \hat{u}_{\mathbb{C}}\right\rangle_{\mathbb{S}}={ }_{\Lambda_{\mathbb{S}}^{\prime}}\left\langle 2 \pi r \lambda_{2}, \hat{u}_{2}-\hat{u}_{3,2}\right\rangle_{\Lambda_{\mathbb{S}}} .
\end{aligned}
$$

In the second duality pairing, $r$ is the radial coordinate ranging in $[0, R]$, being $R$ the radius of the coupling interface $\Gamma$.

\subsection{On the role and choice of the parameter $\alpha$ in (2)}

Variational principle (2) delivers two different solutions for the two different values of $\alpha$, namely 0 and 1 . So $\alpha$ plays a role in defining the way in which the model represents the physical phenomenon we want to address. Generally speaking, when $\alpha=1$ the model ensures the continuity of the value of the field $u$ via the pairing $\langle\cdot, \cdot\rangle_{\mathbb{S}}$ (formally speaking we get $u_{\mathbb{S}}=\mathcal{R}_{\mathbb{S}} u_{\mathbb{C}}$ on $\Gamma_{\mathbb{S}}$ ), whereas it can be shown (see [5]) that the dual variable is continuous in $\Lambda_{\mathbb{C}}^{\prime}$ (formally speaking, $\lambda_{\mathbb{C}}=\mathcal{R}_{\mathbb{S}}^{*} \lambda_{\mathbb{S}}$ on $\Gamma_{\mathbb{C}}$ ). The reciprocal situation occurs when $\alpha=0$, for which the field $u$ is continuous in the sense of the pairing $\langle\cdot, \cdot\rangle_{\mathbb{C}}$, while the flux is continuous in $\Lambda_{\mathbb{S}}^{\prime}$.

The choice of $\alpha$ should be made a priori depending upon the problem that is being addressed. Nevertheless, these two solutions should be close in the sense that both coupled models are addressing the same phenomena. In other words, the quantities of interest retrieved from the computed solutions should not be greatly affected by the choice of the parameter $\alpha$.

At this point, we can distinguish two different kind of situations: either the $\mathbb{C D}$ and the $\mathbb{S D}$ components correspond to real geometrical heterogeneous models, or the 
original problem is geometrically homogeneous and the SD model is a mathematical idealization of the $\mathbb{C D}$ one. In the latter case, if the solutions computed for different values of $\alpha$ are close, then the heterogeneous representation is a good approximation of the originally homogeneous problem.

The choice $\alpha \in(0,1)$ deserves a comment. As noticed above, $\alpha$ provides the way in which the continuity equation is taken into account. Choosing a value of $\alpha \in(0,1)$ would imply that both pairings, and therefore both ways, would be present in the formulation. Notice that in such a case the definition of $\hat{U}_{\mathbb{C}, \mathbb{S}}$ is actually independent of $\alpha$. Due to the inclusion $\Lambda_{\mathbb{S}} \subset \Lambda_{\mathbb{C}}$, we have that the continuity sense in the former is implied by the latter. Therefore, any arbitrary value of $\alpha \in(0,1)$ yields a completely equivalent formulation to that one with $\alpha=0$. For this reason, the cases $\alpha \notin\{0,1\}$ are not meaningful. We can conclude by saying that $\alpha$ plays a physical role more than a mathematical one.

In view of the applications we have in mind it is a better practice to choose the imposition of a weak coupling between the primal variables in the problem, yielding the strong continuity of the dual ones. That is, we want to consider just the pairing $\langle\cdot, \cdot\rangle_{\mathbb{S}}$, which yields the continuity in the space $\Lambda_{\mathbb{S}}$.

It must be highlighted that all the framework that will be presented in what follows can be extended so as to embrace the case $\alpha=0$. This is omitted here for the sake of brevity. Hence, from now on we introduce the following additional assumption.

Assumption 3 We restrict our analysis to the case $\alpha=1$ in (2).

2.6 On the quality of the solution delivered by heterogeneous modeling

The theoretical groundwork provided in the present work is not aimed at evaluating the quality of the solution obtained when modeling a physical system using dimensionallyheterogeneous models. The problem of assessing the quality of the solution in this class of models can be tackled using several approaches, for instance asymptotic analysis as in [13], or using the concept of sensitivity analysis [4].

The basic idea behind the sensitivity analysis is to define a cost functional of interest which represents a meaningful criterion regarding the quality of the solution. As done in [4], the problem can thus be reinterpreted as a problem of shape sensitivity analysis, where the shape change represents the transformation of a 1D description into a 3D model through the displacement of the coupling interface. That is, there is shape change in both models by means of a modification in the position of the underlying boundary surfaces (boundaries $\Gamma_{\mathbb{C}}$ and $\Gamma_{\mathbb{S}}$ in Fig. 2, for instance). Evidently, a compatibility condition in the displacement of these boundary surfaces has to be considered. Then, the calculated sensitivity expressions are capable of quantifying the sensitivity of the cost functional to the position of the coupling interface.

\section{Interface variational formulations}

In this section we rewrite the augmented variational problem (2) in terms of the sole interface variables. Several alternatives will be considered, aimed at the development 
of iterative strategies yielding, at every step, the segregated (i.e., independent) solution of both the complex and simple sub-models.

\subsection{Notational issues and preliminary comments}

Different systems of interface equations can be written according to the way the submodels incorporate the boundary information associated to the interfaces $\Gamma_{\mathbb{C}}$ and $\Gamma_{\mathbb{S}}$. Instances are given by the so-called Neumann-and-Neumann formulation, in which both sub-problems are written in terms of Neumann boundary conditions on the interfaces, or by the Dirichlet-and-Dirichlet system of interface equations in which both sub-problems are formulated using Dirichlet boundary conditions. Several other methods can be derived by suitably combining Dirichlet, Neumann or Robin boundary conditions.

More precisely, when we refer to Neumann, Dirichlet or Robin boundary conditions we are referring always to quantities defined by the SD-model (quantities with index $\mathbb{S})$, which are those chosen to formulate the continuity conditions in the problem. For example, imposing a Dirichlet boundary condition to the $\mathbb{C D}$ models of Sects. 2.3 and 2.4 corresponds to imposing $\mathcal{R}_{\mathbb{S}} u_{\mathbb{C}}$ that is, according to (3) or (5), prescribe that the mean value of $u_{\mathbb{C}}$ is equal to a given $u_{\mathbb{S}}$ on $\Gamma_{\mathbb{C}}$.

Such conditions may be introduced directly in the definition of the functional spaces. Indeed, for $\sigma_{\mathbb{S}} \in \Lambda_{\mathbb{S}}$ we introduce the following linear manifolds

$$
\begin{aligned}
& U_{\mathbb{S}}^{\sigma_{\mathbb{S}}}=\left\{u_{\mathbb{S}} \in U_{\mathbb{S}}: u_{\mathbb{S}}=\sigma_{\mathbb{S}} \text { on } \Gamma_{\mathbb{S}}\right\}, \\
& U_{\mathbb{C}}^{\sigma_{\mathbb{S}}}=\left\{u_{\mathbb{C}} \in U_{\mathbb{C}}: \mathcal{R}_{\mathbb{S}} u_{\mathbb{C}}=\sigma_{\mathbb{S}} \text { on } \Gamma_{\mathbb{S}}\right\}, \\
& \hat{U}_{\mathbb{S}}^{\sigma_{\mathbb{S}}}=\left\{u_{\mathbb{S}} \in \hat{U}_{\mathbb{S}}: u_{\mathbb{S}}=\sigma_{\mathbb{S}} \text { on } \Gamma_{\mathbb{S}}\right\}, \\
& \hat{U}_{\mathbb{C}}^{\sigma_{\mathbb{S}}}=\left\{u_{\mathbb{C}} \in \hat{U}_{\mathbb{C}}: \mathcal{R}_{\mathbb{S}} u_{\mathbb{C}}=\sigma_{\mathbb{S}} \text { on } \Gamma_{\mathbb{S}}\right\} .
\end{aligned}
$$

When $\sigma_{\mathbb{S}}=0$ in (6) above, we obtain the associated linear spaces $\hat{U}_{\mathbb{S}}^{0}$ and $\hat{U}_{\mathbb{C}}^{0}$, and the linear manifolds $U_{\mathbb{S}}^{0}$ and $U_{\mathbb{C}}^{0}$ with homogeneous data on $\Gamma_{\mathbb{S}}$.

This strategy, although possible, is not very convenient in practice. Thus, the approaches based on Lagrange multipliers techniques are preferred, as we will see also in Sect. 3.3.

\subsection{Extension operators for the SD-model}

Consider firstly the operator $\mathcal{D}_{\mathbb{S}}: \Lambda_{\mathbb{S}} \rightarrow \hat{U}_{\mathbb{S}}^{\mu_{\mathbb{S}}}$ defined by the following variational problem: given $\mu_{\mathbb{S}} \in \Lambda_{\mathbb{S}}$, find $\mathcal{D}_{\mathbb{S}} \mu_{\mathbb{S}} \in \hat{U}_{\mathbb{S}}^{\mu_{\mathbb{S}}}$ such that

$$
a_{\mathbb{S}}\left(\mathcal{D}_{\mathbb{S}} \mu_{\mathbb{S}}, \hat{u}_{\mathbb{S}}^{I}\right)=0 \quad \forall \hat{u}_{\mathbb{S}}^{I} \in \hat{U}_{\mathbb{S}}^{0}
$$

It will be used whenever we want to impose a Dirichlet boundary condition on $\Gamma_{\mathbb{S}}$ to the $\mathbb{S D}$-model. 
Another operator we need when imposing a Neumann boundary condition on $\Gamma_{\mathbb{S}}$ to the $\mathbb{S} D$-model is $\mathcal{N}_{\mathbb{S}}: \Lambda_{\mathbb{S}}^{\prime} \rightarrow \hat{U}_{\mathbb{S}}$ defined by the following variational problem: given $\lambda_{\mathbb{S}} \in \Lambda_{\mathbb{S}}^{\prime}$, find $\mathcal{N}_{\mathbb{S}} \lambda_{\mathbb{S}} \in \hat{U}_{\mathbb{S}}$ such that

$$
a_{\mathbb{S}}\left(\mathcal{N}_{\mathbb{S}} \lambda_{\mathbb{S}}, \hat{u}_{\mathbb{S}}^{J}\right)=-\left\langle\lambda_{\mathbb{S}}, \hat{u}_{\mathbb{S}}^{J}\right\rangle_{\mathbb{S}} \quad \forall \hat{u}_{\mathbb{S}}^{J} \in \hat{U}_{\mathbb{S}}
$$

The Lax-Milgram theorem (see, e.g., [17]) guarantees straightforwardly the wellposedness of problem (7) and the existence of $\mathcal{N}_{\mathbb{S}} \lambda_{\mathbb{S}}$ in (8). The uniqueness of $\mathcal{N}_{\mathbb{S}} \lambda_{\mathbb{S}}$ might be guaranteed up to an additive constant depending on the boundary conditions imposed on $\partial \Omega_{\mathbb{S}} \backslash \Gamma_{\mathbb{S}}$. Moreover, in case a Neumann boundary condition is assigned on $\partial \Omega_{\mathbb{S}} \backslash \Gamma_{\mathbb{S}}$, special attention must be paid in choosing the extension $\mathcal{N}_{\mathbb{S}} \lambda_{\mathbb{S}}$ so to satisfy the compatibility condition required by a full-Neumann problem.

\subsection{Extension operators for the $\mathbb{C D}$-model}

We proceed similarly for the $\mathbb{C D}$-model by defining the operator $\mathcal{D}_{\mathbb{C}}: \Lambda_{\mathbb{S}} \rightarrow \hat{U}_{\mathbb{C}}^{\mu_{\mathbb{S}}}$ as follows: given $\mu_{\mathbb{S}} \in \Lambda_{\mathbb{S}}$, find $\mathcal{D}_{\mathbb{C}} \mu_{\mathbb{S}} \in \hat{U}_{\mathbb{C}}^{\mu_{\mathbb{S}}}$ such that

$$
a_{\mathbb{C}}\left(\mathcal{D}_{\mathbb{C}} \mu_{\mathbb{S}}, \hat{u}_{\mathbb{C}}^{I}\right)=0 \quad \forall \hat{u}_{\mathbb{C}}^{I} \in \hat{U}_{\mathbb{C}}^{0}
$$

This operator imposes Dirichlet boundary conditions on $\Gamma_{\mathbb{C}}$, which amounts to impose the value of $\mathcal{R}_{\mathbb{S}} u_{\mathbb{C}}$ in this context.

The weak formulation (9) can be equivalently rewritten by using Lagrange multipliers to impose the condition $\mathcal{R}_{\mathbb{S}}\left(\mathcal{D}_{\mathbb{C}} \mu_{\mathbb{S}}\right)=\mu_{\mathbb{S}}$ on $\Gamma_{\mathbb{S}}$, which is fulfilled by the elements of $\hat{U}_{\mathbb{C}}^{\mu_{\mathbb{S}}}$, as follows: find $\left(\mathcal{D}_{\mathbb{C}} \mu_{\mathbb{S}}, \lambda_{\mathbb{S}}\right) \in \hat{U}_{\mathbb{C}} \times \Lambda_{\mathbb{S}}^{\prime}$ such that

$$
\begin{aligned}
a_{\mathbb{C}}\left(\mathcal{D}_{\mathbb{C}} \mu_{\mathbb{S}}, \hat{u}_{\mathbb{C}}^{I}\right)+\left\langle\mathcal{R}_{\mathbb{S}}^{*} \lambda_{\mathbb{S}}, \hat{u}_{\mathbb{C}}^{I}\right\rangle_{\mathbb{C}}=0 \quad \forall \hat{u}_{\mathbb{C}}^{I} \in \hat{U}_{\mathbb{C}} \\
\left\langle\mathcal{R}_{\mathbb{S}}^{*} \hat{\lambda}_{\mathbb{S}}, \mathcal{D}_{\mathbb{C}} \mu_{\mathbb{S}}\right\rangle_{\mathbb{C}}=\left\langle\hat{\lambda}_{\mathbb{S}}, \mu_{\mathbb{S}}\right\rangle_{\mathbb{S}} \quad \forall \hat{\lambda}_{\mathbb{S}} \in \Lambda_{\mathbb{S}}^{\prime}
\end{aligned}
$$

Finally, to impose a Neumann boundary condition to the $\mathbb{C D}$-model we need the operator $\mathcal{N}_{\mathbb{C}}: \Lambda_{\mathbb{S}}^{\prime} \rightarrow \hat{U}_{\mathbb{C}}$ s.t. for any given $\lambda_{\mathbb{S}} \in \Lambda_{\mathbb{S}}^{\prime}, \mathcal{N}_{\mathbb{C}} \lambda_{\mathbb{S}} \in \hat{U}_{\mathbb{C}}$ satisfies

$$
a_{\mathbb{C}}\left(\mathcal{N}_{\mathbb{C}} \lambda_{\mathbb{S}}, \hat{u}_{\mathbb{C}}^{J}\right)=\left\langle\mathcal{R}_{\mathbb{S}}^{*} \lambda_{\mathbb{S}}, \hat{u}_{\mathbb{C}}^{J}\right\rangle_{\mathbb{C}}=\left\langle\lambda_{\mathbb{S}}, \mathcal{R}_{\mathbb{S}} \hat{u}_{\mathbb{C}}^{J}\right\rangle_{\mathbb{S}} \quad \forall \hat{u}_{\mathbb{C}}^{J} \in \hat{U}_{\mathbb{C}}
$$

where the right hand side is consistent with the duality pairings seen in (2) for $\alpha=1$.

About the well-posedness of problems (10) and (11), we can prove the following result.

Proposition 1 If the adjoint operator $\mathcal{R}_{\mathbb{S}}^{*}: \Lambda_{\mathbb{S}}^{\prime} \rightarrow \Lambda_{\mathbb{C}}^{\prime}$ is linear and there exist two constants $0<C_{1}<C_{2}<\infty$ such that

$$
C_{1}\left\|\hat{\lambda}_{\mathbb{S}}\right\|_{\Lambda_{\mathbb{S}}^{\prime}} \leq\left\|\mathcal{R}_{\mathbb{S}}^{*} \hat{\lambda}_{\mathbb{S}}\right\|_{\Lambda_{\mathbb{C}}^{\prime}} \leq C_{2}\left\|\hat{\lambda}_{\mathbb{S}}\right\|_{\Lambda_{\mathbb{S}}^{\prime}} \quad \forall \hat{\lambda}_{\mathbb{S}} \in \Lambda_{\mathbb{S}}^{\prime}
$$


then problem (10) is well-posed. Moreover, the operator $\mathcal{D}_{\mathbb{C}}$ is continuous, i.e. there exists a constant $C_{3}>0$ such that

$$
\left\|\mathcal{D}_{\mathbb{C}} \mu_{\mathbb{S}}\right\|_{U_{\mathbb{C}}} \leq C_{3}\left\|\mu_{\mathbb{S}}\right\|_{\Lambda_{\mathbb{S}}} \quad \forall \mu_{\mathbb{S}} \in \Lambda_{\mathbb{S}}
$$

Proof The proof follows the guidelines of Theorem 3.1 in [1].

We introduce the Hilbert space $H=\hat{U}_{\mathbb{C}} \times \Lambda_{\mathbb{S}}^{\prime}$ with norm $\left\|\left(u_{\mathbb{C}}, \lambda_{\mathbb{S}}\right)\right\|_{H}^{2}=\left\|u_{\mathbb{C}}\right\|_{\hat{U}_{\mathbb{C}}}^{2}+$ $\left\|\lambda_{\mathbb{S}}\right\|_{\Lambda_{\mathbb{S}}^{\prime}}^{2}$ and the bilinear symmetric form:

$$
B\left(u_{\mathbb{C}}, \lambda_{\mathbb{S}} ; v_{\mathbb{C}}, \xi_{\mathbb{S}}\right)=a_{\mathbb{C}}\left(u_{\mathbb{C}}, v_{\mathbb{C}}\right)+\left\langle\mathcal{R}_{\mathbb{S}}^{*} \lambda_{\mathbb{S}}, v_{\mathbb{C}}\right\rangle_{\mathbb{C}}+\left\langle\mathcal{R}_{\mathbb{S}}^{*} \xi_{\mathbb{S}}, u_{\mathbb{C}}\right\rangle_{\mathbb{C}}
$$

for all $\left(u_{\mathbb{C}}, \lambda_{\mathbb{S}}\right),\left(v_{\mathbb{C}}, \xi_{\mathbb{S}}\right) \in H$.

Since the bilinear form $a_{\mathbb{C}}(\cdot, \cdot)$ is continuous in $\hat{U}_{\mathbb{C}} \times \hat{U}_{\mathbb{C}}$ and there is a continuous mapping $\hat{U}_{\mathbb{C}} \hookrightarrow \Lambda_{\mathbb{C}}$, using (12) we have that also $B$ is continuous:

$$
\left|B\left(u_{\mathbb{C}}, \lambda_{\mathbb{S}} ; v_{\mathbb{C}}, \xi_{\mathbb{S}}\right)\right| \leq C\left\|\left(u_{\mathbb{C}}, \lambda_{\mathbb{S}}\right)\right\|_{H}\left\|\left(v_{\mathbb{C}}, \xi_{\mathbb{S}}\right)\right\|_{H} \quad \forall\left(u_{\mathbb{C}}, \lambda_{\mathbb{S}}\right),\left(v_{\mathbb{C}}, \xi_{\mathbb{S}}\right) \in H
$$

Now, denoting $w_{\mathbb{C}} \in \hat{U}_{\mathbb{C}}$ the solution of the following problem

$$
a_{\mathbb{C}}\left(w_{\mathbb{C}}, v_{\mathbb{C}}\right)=\left\langle\mathcal{R}_{\mathbb{S}}^{*} \lambda_{\mathbb{S}}, v_{\mathbb{C}}\right\rangle_{\mathbb{C}} \quad \forall v_{\mathbb{C}} \in \hat{U}_{\mathbb{C}}
$$

we can proceed like in Theorem 3.1 in [1] and obtain that there exists a constant $C>0$ s.t. for any given $\left(u_{\mathbb{C}}, \lambda_{\mathbb{S}}\right) \in H$,

$$
\sup _{\substack{\left(v_{\mathbb{C}}, \xi_{\mathbb{S}}\right) \in H \\\left(v_{\mathbb{C}}, \xi_{\mathbb{S}}\right) \neq 0}} \frac{\left|B\left(u_{\mathbb{C}}, \lambda_{\mathbb{S}} ; v_{\mathbb{C}}, \xi_{\mathbb{S}}\right)\right|}{\left\|\left(v_{\mathbb{C}}, \xi_{\mathbb{S}}\right)\right\|_{H}} \geq C\left\|\left(u_{\mathbb{C}}, \lambda_{\mathbb{S}}\right)\right\|_{H}
$$

Notice that to obtain this result we have used the hypothesis (12) and the inequalities $\left\|w_{\mathbb{C}}\right\|_{\hat{U}_{\mathbb{C}}} \leq C\left\|\mathcal{R}_{\mathbb{S}}^{*} \lambda_{\mathbb{S}}\right\|_{\Lambda_{\mathbb{C}}^{\prime}}$ and $C\left\|\mathcal{R}_{\mathbb{S}}^{*} \lambda_{\mathbb{S}}\right\|_{\Lambda_{\mathbb{C}}^{\prime}}^{2} \leq\left\langle\mathcal{R}_{\mathbb{S}}^{*} \lambda_{\mathbb{S}}, w_{\mathbb{C}}\right\rangle_{\mathbb{C}}$

Thanks to Theorem 2.8 in [1] we can conclude that the weak problem (10) has a unique solution and that (13) holds.

In turn, the well-posedness of (11) is a consequence of the Lax-Milgram theorem and of the continuity of the adjoint operator $\mathcal{R}_{\mathbb{S}}^{*}$. As in problem (8), notice that the solution might be unique up to an additive constant depending on the boundary conditions imposed on $\partial \Omega_{\mathbb{C}} \backslash \Gamma_{\mathbb{C}}$. Finally, remark that the extension $\mathcal{N}_{\mathbb{C}} \lambda_{\mathbb{S}}$ must fulfill a compatibility condition if Neumann boundary conditions are imposed also on $\partial \Omega_{\mathbb{C}} \backslash \Gamma_{\mathbb{C}}$.

Remark 2 Consider the 3D-1D example seen in Sect. 2.3. The operator $\mathcal{R}_{\mathbb{S}}$ provides the mean value over $\Gamma$ of a function in $H^{1 / 2}(\Gamma)$. As seen in (4), the duality $\Lambda_{\mathbb{S}} \times \Lambda_{\mathbb{S}}^{\prime}$ 
is written as

$$
\left\langle\lambda_{\mathbb{S}}, \mathcal{R}_{\mathbb{S}} \hat{u}_{\mathbb{C}}\right\rangle_{\mathbb{S}}=\underbrace{\lambda_{1}}_{\in \mathbb{R}} \underbrace{\left(\int_{\Gamma} u_{3} \mathrm{~d} \Gamma\right)}_{\in \mathbb{R}}={ }_{H^{-1 / 2}(\Gamma)}\left\langle\mathcal{R}_{\mathbb{S}}^{*} \lambda_{1}, u_{3}\right\rangle_{H^{1 / 2}(\Gamma)}=\left\langle\mathcal{R}_{\mathbb{S}}^{*} \lambda_{\mathbb{S}}, \hat{u}_{\mathbb{C}}\right\rangle_{\mathbb{C}}
$$

We see that in the present case, for a given real number, the operator $\mathcal{R}_{\mathbb{S}}^{*} \lambda_{\mathbb{S}}$ gives the extension as a constant function defined in all $\Gamma$. This operator satisfies the hypotheses of Proposition 1.

\subsection{Steklov-Poincaré formulation (one unknown)}

To reformulate (2) as a Steklov-Poincaré interface equation, we proceed as follows. At first, we consider the following decompositions

$$
u_{\mathbb{S}}=u_{\mathbb{S}}^{I}+\mathcal{D}_{\mathbb{S}} \mu_{\mathbb{S}}, \quad u_{\mathbb{C}}=u_{\mathbb{C}}^{I}+\mathcal{D}_{\mathbb{C}} \mu_{\mathbb{S}},
$$

where the extension operators $\mathcal{D}_{\mathbb{S}}$ and $\mathcal{D}_{\mathbb{C}}$ were defined in (7) and (9), respectively. The functions $u_{\mathbb{S}}^{I} \in U_{\mathbb{S}}^{0}$ and $u_{\mathbb{C}}^{I} \in U_{\mathbb{C}}^{0}$ (see Eq. (6) for the definition of these affine manifolds) are the solutions of the following problems

$$
\begin{aligned}
a_{\mathbb{S}}\left(u_{\mathbb{S}}^{I}, \hat{u}_{\mathbb{S}}^{I}\right)=f_{\mathbb{S}}\left(\hat{u}_{\mathbb{S}}^{I}\right) & \forall \hat{u}_{\mathbb{S}}^{I} \in \hat{U}_{\mathbb{S}}^{0}, \\
a_{\mathbb{C}}\left(u_{\mathbb{C}}^{I}, \hat{u}_{\mathbb{C}}^{I}\right)=f_{\mathbb{C}}\left(\hat{u}_{\mathbb{C}}^{I}\right) & \forall \hat{u}_{\mathbb{C}}^{I} \in \hat{U}_{\mathbb{C}}^{0}
\end{aligned}
$$

Correspondingly, the variations (test functions) $\hat{u}_{\mathbb{S}}$ and $\hat{u}_{\mathbb{C}}$ in (2) are split as follows

$$
\begin{aligned}
& \hat{u}_{\mathbb{S}}=\hat{u}_{\mathbb{S}}^{I}+\widehat{\mathcal{D}_{\mathbb{S}} \mu_{\mathbb{S}}}=\hat{u}_{\mathbb{S}}^{I}+\mathcal{D}_{\mathbb{S}} \hat{\mu}_{\mathbb{S}} \\
& \hat{u}_{\mathbb{C}}=\hat{u}_{\mathbb{C}}^{I}+\widehat{\mathcal{D}_{\mathbb{C}} \mu_{\mathbb{S}}}=\hat{u}_{\mathbb{C}}^{I}+\mathcal{D}_{\mathbb{C}} \hat{\mu}_{\mathbb{S}}
\end{aligned}
$$

with $\hat{u}_{\mathbb{S}}=\hat{\mu}_{\mathbb{S}}$ and $\mathcal{R}_{\mathbb{S}} \hat{u}_{\mathbb{C}}=\hat{\mu}_{\mathbb{S}}$ on $\Gamma_{\mathbb{S}}$. With the previous definitions and using (15) and (17) into (2) (for $\alpha=1$ ) we have the following equivalent problem: given $u_{\mathbb{S}}^{I}$ and $u_{\mathbb{C}}^{I}$ solutions of (16), find $\mu_{\mathbb{S}} \in \Lambda_{\mathbb{S}}$ such that

$$
\begin{gathered}
a_{\mathbb{S}}\left(u_{\mathbb{S}}^{I}+\mathcal{D}_{\mathbb{S}} \mu_{\mathbb{S}}, \hat{u}_{\mathbb{S}}^{I}+\mathcal{D}_{\mathbb{S}} \hat{\mu}_{\mathbb{S}}\right)+a_{\mathbb{C}}\left(u_{\mathbb{C}}^{I}+\mathcal{D}_{\mathbb{C}} \mu_{\mathbb{S}}, \hat{u}_{\mathbb{C}}^{I}+\mathcal{D}_{\mathbb{C}} \hat{\mu}_{\mathbb{S}}\right) \\
=f_{\mathbb{S}}\left(\hat{u}_{\mathbb{S}}^{I}+\mathcal{D}_{\mathbb{S}} \hat{\mu}_{\mathbb{S}}\right)+f_{\mathbb{C}}\left(\hat{u}_{\mathbb{C}}^{I}+\mathcal{D}_{\mathbb{C}} \hat{\mu}_{\mathbb{S}}\right) \quad \forall \hat{\mu}_{\mathbb{S}} \in \Lambda_{\mathbb{S}} .
\end{gathered}
$$


By rearranging the terms we obtain

$$
\begin{aligned}
& \underbrace{a_{\mathbb{S}}\left(u_{\mathbb{S}}^{I}, \hat{u}_{\mathbb{S}}^{I}\right)-f_{\mathbb{S}}\left(\hat{u}_{\mathbb{S}}^{I}\right)}_{=0 \text { by }(16)}+a_{\mathbb{S}}\left(u_{\mathbb{S}}^{I}, \mathcal{D}_{\mathbb{S}} \hat{\mu}_{\mathbb{S}}\right)+\underbrace{a_{\mathbb{S}}\left(\mathcal{D}_{\mathbb{S}} \mu_{\mathbb{S}}, \hat{u}_{\mathbb{S}}^{I}\right)}_{=0 \text { by }(7)}+a_{\mathbb{S}}\left(\mathcal{D}_{\mathbb{S}} \mu_{\mathbb{S}}, \mathcal{D}_{\mathbb{S}} \hat{\mu}_{\mathbb{S}}\right) \\
& \quad+\underbrace{a_{\mathbb{C}}\left(u_{\mathbb{C}}^{I}, \hat{u}_{\mathbb{C}}^{I}\right)-f_{\mathbb{C}}\left(\hat{u}_{\mathbb{C}}^{I}\right)}_{=0 \text { by }(16)}+a_{\mathbb{C}}\left(u_{\mathbb{C}}^{I}, \mathcal{D}_{\mathbb{C}} \hat{\mu}_{\mathbb{S}}\right)+\underbrace{a_{\mathbb{C}}\left(\mathcal{D}_{\mathbb{C}} \mu_{\mathbb{S}}, \hat{u}_{\mathbb{C}}^{I}\right)}_{=0 \text { by }(9)}+a_{\mathbb{C}}\left(\mathcal{D}_{\mathbb{C}} \mu_{\mathbb{S}}, \mathcal{D}_{\mathbb{C}} \hat{\mu}_{\mathbb{S}}\right) \\
& =f_{\mathbb{S}}\left(\mathcal{D}_{\mathbb{S}} \hat{\mu}_{\mathbb{S}}\right)+f_{\mathbb{C}}\left(\mathcal{D}_{\mathbb{C}} \hat{\mu}_{\mathbb{S}}\right) \quad \forall \hat{\mu}_{\mathbb{S}} \in \Lambda_{\mathbb{S}} .
\end{aligned}
$$

In summary, we find the following Steklov-Poincaré reformulation of (2): given $u_{\mathbb{S}}^{I}$ and $u_{\mathbb{C}}^{I}$ solutions of (16), find $\mu_{\mathbb{S}} \in \Lambda_{\mathbb{S}}$ such that

$$
\begin{aligned}
& a_{\mathbb{S}}\left(\mathcal{D}_{\mathbb{S}} \mu_{\mathbb{S}}, \mathcal{D}_{\mathbb{S}} \hat{\mu}_{\mathbb{S}}\right)+a_{\mathbb{C}}\left(\mathcal{D}_{\mathbb{C}} \mu_{\mathbb{S}}, \mathcal{D}_{\mathbb{C}} \hat{\mu}_{\mathbb{S}}\right)=f_{\mathbb{S}}\left(\mathcal{D}_{\mathbb{S}} \hat{\mu}_{\mathbb{S}}\right)-a_{\mathbb{S}}\left(u_{\mathbb{S}}^{I}, \mathcal{D}_{\mathbb{S}} \hat{\mu}_{\mathbb{S}}\right) \\
& +f_{\mathbb{C}}\left(\mathcal{D}_{\mathbb{C}} \hat{\mu}_{\mathbb{S}}\right)-a_{\mathbb{C}}\left(u_{\mathbb{C}}^{I}, \mathcal{D}_{\mathbb{C}} \hat{\mu}_{\mathbb{S}}\right) \quad \forall \hat{\mu}_{\mathbb{S}} \in \Lambda_{\mathbb{S}}
\end{aligned}
$$

or, in compact form,

$$
s_{\Gamma_{\mathbb{S}}}\left(\mu_{\mathbb{S}}, \hat{\mu}_{\mathbb{S}}\right)=g_{\Gamma_{\mathbb{S}}}\left(\hat{\mu}_{\mathbb{S}}\right) \quad \forall \hat{\mu}_{\mathbb{S}} \in \Lambda_{\mathbb{S}}
$$

where the bilinear form $s_{\Gamma_{\mathbb{S}}}: \Lambda_{\mathbb{S}} \times \Lambda_{\mathbb{S}} \rightarrow \mathbb{R}$ and the linear form $g_{\Gamma_{\mathbb{S}}}: \Lambda_{\mathbb{S}} \rightarrow \mathbb{R}$ are respectively given by

$$
\begin{aligned}
s_{\Gamma_{\mathbb{S}}}\left(\mu_{\mathbb{S}}, \hat{\mu}_{\mathbb{S}}\right) & =a_{\mathbb{S}}\left(\mathcal{D}_{\mathbb{S}} \mu_{\mathbb{S}}, \mathcal{D}_{\mathbb{S}} \hat{\mu}_{\mathbb{S}}\right)+a_{\mathbb{C}}\left(\mathcal{D}_{\mathbb{C}} \mu_{\mathbb{S}}, \mathcal{D}_{\mathbb{C}} \hat{\mu}_{\mathbb{S}}\right) \\
g_{\Gamma_{\mathbb{S}}}\left(\hat{\mu}_{\mathbb{S}}\right) & =f_{\mathbb{S}}\left(\mathcal{D}_{\mathbb{S}} \hat{\mu}_{\mathbb{S}}\right)-a_{\mathbb{S}}\left(u_{\mathbb{S}}^{I}, \mathcal{D}_{\mathbb{S}} \hat{\mu}_{\mathbb{S}}\right)+f_{\mathbb{C}}\left(\mathcal{D}_{\mathbb{C}} \hat{\mu}_{\mathbb{S}}\right)-a_{\mathbb{C}}\left(u_{\mathbb{C}}^{I}, \mathcal{D}_{\mathbb{C}} \hat{\mu}_{\mathbb{S}}\right) .
\end{aligned}
$$

In operator form (19) reads as follows

$$
\mathcal{S}_{\Gamma_{\mathbb{S}}} \mu_{\mathbb{S}}=g_{\Gamma_{\mathbb{S}}} \quad \text { in } \Lambda_{\mathbb{S}}^{\prime}
$$

with obvious choice of notations.

Remark 3 When coupling 3D and 1D models like in Sect. 2.3 the variational Eq. (20) reduces to a scalar equation with one unknown

$$
S \mu=g \quad \text { in } \mathbb{R},
$$

where $S$ and $g$ are real numbers. In this case the problem is of dimension 1.

Proposition 2 There exists a unique solution $\mu_{\mathbb{S}} \in \Lambda_{\mathbb{S}}$ of (19). Moreover, there exists $C>0$ such that the solution satisfies

$$
\left\|\mu_{\mathbb{S}}\right\|_{\Lambda_{\mathbb{S}}} \leq C\left\|g_{\Gamma_{\mathbb{S}}}\right\|_{\Lambda_{\mathbb{S}}^{\prime}}
$$


Proof From the bilinearity and continuity of $a_{\mathbb{S}}(\cdot, \cdot)$ and $a_{\mathbb{C}}(\cdot, \cdot)$ and the continuity of the operators $\mathcal{D}_{\mathbb{S}}$ and $\mathcal{D}_{\mathbb{C}}$ it follows that $s_{\Gamma_{\mathbb{S}}}$ is also continuous, that is, there exists $\beta>0$ such that

$$
\left|s_{\Gamma_{\mathbb{S}}}\left(\mu_{\mathbb{S}}, \eta_{\mathbb{S}}\right)\right| \leq \beta\left\|\mu_{\mathbb{S}}\right\|_{\Lambda_{\mathbb{S}}}\left\|\eta_{\mathbb{S}}\right\|_{\Lambda_{\mathbb{S}}} \quad \forall \mu_{\mathbb{S}}, \eta_{\mathbb{S}} \in \Lambda_{\mathbb{S}}
$$

Using similar arguments we have that $g_{\Gamma_{\mathbb{S}}}$ is continuous, that is, there exists $\gamma>0$ such that

$$
\left|g_{\Gamma_{\mathbb{S}}}\left(\eta_{\mathbb{S}}\right)\right| \leq \gamma\left\|\eta_{\mathbb{S}}\right\|_{\Lambda_{\mathbb{S}}} \quad \forall \eta \in \Lambda_{\mathbb{S}}
$$

Also, from the coercivity of $a_{\mathbb{S}}(\cdot, \cdot)$ and $a_{\mathbb{C}}(\cdot, \cdot)$ and from the trace inequalities it follows that $s_{\Gamma_{\mathbb{S}}}$ is coercive, that is, there exists $\alpha>0$ such that

$$
s_{\Gamma_{\mathbb{S}}}\left(\mu_{\mathbb{S}}, \mu_{\mathbb{S}}\right) \geq \alpha\left\|\mu_{\mathbb{S}}\right\|_{\Lambda_{\mathbb{S}}}^{2} \quad \forall \mu_{\mathbb{S}} \in \Lambda_{\mathbb{S}}
$$

Thus, the existence and uniqueness of the solution $\mu_{\mathbb{S}} \in \Lambda_{\mathbb{S}}$ is guaranteed by the Lax-Milgram theorem, and estimate (21) holds as a corollary.

\subsection{Augmented formulation (two unknowns)}

In Sect. 3.4 the variational problem (2) was recasted into a variational interface problem depending on the single interface unknown $\mu_{\mathbb{S}}$. Here we rewrite the same problem in terms of two variables, $\mu_{\mathbb{S}}$ and $\lambda_{\mathbb{S}}$ (primal and dual). We present three different (equivalent) strategies. The denomination in each case will be clear from the context and follows the comments made in Sect. 3.1.

Remark 4 Within the present framework it will be possible to select quite arbitrarily the interface conditions to be imposed at both models arriving at a given coupling interface. In other words, since we are keeping both variables $\mu_{\mathbb{S}}$ and $\lambda_{\mathbb{S}}$ we can independently set different interface conditions for both models sharing the same coupling interface.

\subsubsection{Approach 1: Dirichlet-and-Dirichlet decomposition}

Let us consider the decomposition of $u_{\mathbb{S}}$ and $u_{\mathbb{C}}$ as in (15), with $\mathcal{D}_{\mathbb{S}} \mu_{\mathbb{S}} \in \hat{U}_{\mathbb{S}}^{\mu_{\mathbb{S}}}$ and $\mathcal{D}_{\mathbb{C}} \mu_{\mathbb{S}} \in \hat{U}_{\mathbb{C}}^{\mu_{\mathbb{S}}}$ satisfying (7) and (9), and $u_{\mathbb{S}}^{I} \in U_{\mathbb{S}}^{0}$ and $u_{\mathbb{C}}^{I} \in U_{\mathbb{C}}^{0}$ satisfying (16). The denomination Dirichlet-and-Dirichlet decomposition stems from the fact that $u_{\mathbb{S}}$ and $u_{\mathbb{C}}$ are decomposed through contributions which are defined via Dirichlet subproblems for both the $\mathbb{S D}$-model and the $\mathbb{C D}$-model.

However, instead of (17) we consider

$$
\hat{u}_{\mathbb{S}}=\hat{u}_{\mathbb{S}}^{I}+\mathcal{D}_{\mathbb{S}} \hat{\mu}_{\mathbb{S}}^{1}, \quad \hat{u}_{\mathbb{C}}=\hat{u}_{\mathbb{C}}^{I}+\mathcal{D}_{\mathbb{C}} \hat{\mu}_{\mathbb{S}}^{2}
$$


Now, contrariwise to (17), it is $\hat{\mu}_{\mathbb{S}}^{1} \neq \hat{\mu}_{\mathbb{S}}^{2}$. Hence, we rewrite the variational problem (2) as follows: given $u_{\mathbb{S}}^{I}$ and $u_{\mathbb{C}}^{I}$ solutions of (16), find $\left(\mu_{\mathbb{S}}, \lambda_{\mathbb{S}}\right) \in \Lambda_{\mathbb{S}} \times \Lambda_{\mathbb{S}}^{\prime}$ such that

$$
\begin{aligned}
a_{\mathbb{S}} & \left(u_{\mathbb{S}}^{I}+\mathcal{D}_{\mathbb{S}} \mu_{\mathbb{S}}, \hat{u}_{\mathbb{S}}^{I}+\mathcal{D}_{\mathbb{S}} \hat{\mu}_{\mathbb{S}}^{1}\right)+a_{\mathbb{C}}\left(u_{\mathbb{C}}^{I}+\mathcal{D}_{\mathbb{C}} \mu_{\mathbb{S}}, \hat{u}_{\mathbb{C}}^{I}+\mathcal{D}_{\mathbb{C}} \hat{\mu}_{\mathbb{S}}^{2}\right) \\
& +\left\langle\lambda_{\mathbb{S}}, \hat{u}_{\mathbb{S}}^{I}-\mathcal{R}_{\mathbb{S}} \hat{u}_{\mathbb{C}}^{I}\right\rangle_{\mathbb{S}}+\left\langle\lambda_{\mathbb{S}}, \hat{\mu}_{\mathbb{S}}^{1}-\hat{\mu}_{\mathbb{S}}^{2}\right\rangle_{\mathbb{S}} \\
= & f_{\mathbb{S}}\left(\hat{u}_{\mathbb{S}}^{I}+\mathcal{D}_{\mathbb{S}} \hat{\mu}_{\mathbb{S}}^{1}\right)+f_{\mathbb{C}}\left(\hat{u}_{\mathbb{C}}^{I}+\mathcal{D}_{\mathbb{C}} \hat{\mu}_{\mathbb{S}}^{2}\right) \quad \forall\left(\hat{\mu}_{\mathbb{S}}^{1}, \hat{\mu}_{\mathbb{S}}^{2}\right) \in \Lambda_{\mathbb{S}} \times \Lambda_{\mathbb{S}} .
\end{aligned}
$$

After rearranging some terms and using (7), (9) and (16) as in (18) we obtain

$$
\begin{aligned}
& a_{\mathbb{S}}\left(\mathcal{D}_{\mathbb{S}} \mu_{\mathbb{S}}, \mathcal{D}_{\mathbb{S}} \hat{\mu}_{\mathbb{S}}^{1}\right)+a_{\mathbb{C}}\left(\mathcal{D}_{\mathbb{C}} \mu_{\mathbb{S}}, \mathcal{D}_{\mathbb{C}} \hat{\mu}_{\mathbb{S}}^{2}\right)+\left\langle\lambda_{\mathbb{S}}, \hat{\mu}_{\mathbb{S}}^{1}-\hat{\mu}_{\mathbb{S}}^{2}\right\rangle_{\mathbb{S}} \\
& =f_{\mathbb{S}}\left(\mathcal{D}_{\mathbb{S}} \hat{\mu}_{\mathbb{S}}^{1}\right)-a_{\mathbb{S}}\left(u_{\mathbb{S}}^{I}, \mathcal{D}_{\mathbb{S}} \hat{\mu}_{\mathbb{S}}^{1}\right) \\
& \quad+f_{\mathbb{C}}\left(\mathcal{D}_{\mathbb{C}} \hat{\mu}_{\mathbb{S}}^{2}\right)-a_{\mathbb{C}}\left(u_{\mathbb{C}}^{I}, \mathcal{D}_{\mathbb{C}} \hat{\mu}_{\mathbb{S}}^{2}\right) \quad \forall\left(\hat{\mu}_{\mathbb{S}}^{1}, \hat{\mu}_{\mathbb{S}}^{2}\right) \in \Lambda_{\mathbb{S}} \times \Lambda_{\mathbb{S}},
\end{aligned}
$$

that is find $\left(\mu_{\mathbb{S}}, \lambda_{\mathbb{S}}\right) \in \Lambda_{\mathbb{S}} \times \Lambda_{\mathbb{S}}^{\prime}$ such that

$$
\begin{array}{ll}
s_{\Gamma_{\mathbb{S}}, \mathbb{S}}\left(\mu_{\mathbb{S}}, \hat{\mu}_{\mathbb{S}}^{1}\right)+\left\langle\lambda_{\mathbb{S}}, \hat{\mu}_{\mathbb{S}}^{1}\right\rangle_{\mathbb{S}}=g_{\Gamma_{\mathbb{S}}, \mathbb{S}}\left(\hat{\mu}_{\mathbb{S}}^{1}\right) & \forall \hat{\mu}_{\mathbb{S}}^{1} \in \Lambda_{\mathbb{S}}, \\
s_{\Gamma_{\mathbb{S}}, \mathbb{C}}\left(\mu_{\mathbb{S}}, \hat{\mu}_{\mathbb{S}}^{2}\right)-\left\langle\lambda_{\mathbb{S}}, \hat{\mu}_{\mathbb{S}}^{2}\right\rangle_{\mathbb{S}}=g_{\Gamma_{\mathbb{S}}, \mathbb{C}}\left(\hat{\mu}_{\mathbb{S}}^{2}\right) & \forall \hat{\mu}_{\mathbb{S}}^{2} \in \Lambda_{\mathbb{S}} .
\end{array}
$$

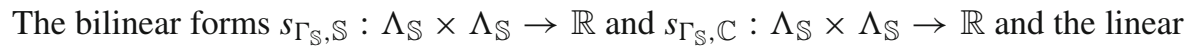
forms $g_{\Gamma_{\mathbb{S}}, \mathbb{S}}: \Lambda_{\mathbb{S}} \rightarrow \mathbb{R}$ and $g_{\Gamma_{\mathbb{S}}, \mathbb{C}}: \Lambda_{\mathbb{S}} \rightarrow \mathbb{R}$ are given by

$$
\begin{aligned}
s_{\Gamma_{\mathbb{S}}, \mathbb{S}}\left(\mu_{\mathbb{S}}, \hat{\mu}_{\mathbb{S}}^{1}\right) & =a_{\mathbb{S}}\left(\mathcal{D}_{\mathbb{S}} \mu_{\mathbb{S}}, \mathcal{D}_{\mathbb{S}} \hat{\mu}_{\mathbb{S}}^{1}\right), \\
s_{\Gamma_{\mathbb{S}}, \mathbb{C}}\left(\mu_{\mathbb{S}}, \hat{\mu}_{\mathbb{S}}^{2}\right) & =a_{\mathbb{C}}\left(\mathcal{D}_{\mathbb{C}} \mu_{\mathbb{S}}, \mathcal{D}_{\mathbb{C}} \hat{\mu}_{\mathbb{S}}^{2}\right), \\
g_{\Gamma_{\mathbb{S}}, \mathbb{S}}\left(\hat{\mu}_{\mathbb{S}}^{1}\right) & =f_{\mathbb{S}}\left(\mathcal{D}_{\mathbb{S}} \hat{\mu}_{\mathbb{S}}^{1}\right)-a_{\mathbb{S}}\left(u_{\mathbb{S}}^{I}, \mathcal{D}_{\mathbb{S}} \hat{\mu}_{\mathbb{S}}^{1}\right), \\
g_{\Gamma_{\mathbb{S}}, \mathbb{C}}\left(\hat{\mu}_{\mathbb{S}}^{2}\right) & =f_{\mathbb{C}}\left(\mathcal{D}_{\mathbb{C}} \hat{\mu}_{\mathbb{S}}^{2}\right)-a_{\mathbb{C}}\left(u_{\mathbb{C}}^{I}, \mathcal{D}_{\mathbb{C}} \hat{\mu}_{\mathbb{S}}^{2}\right) .
\end{aligned}
$$

From (23) we can derive (19) easily, by adding (23) 1 and (23) 2 and taking $\hat{\mu}_{\mathbb{S}}^{1}=\hat{\mu}_{\mathbb{S}}^{2}=$ $\hat{\mu}_{\mathbb{S}}$. As done for (20), we can write (23) in a more compact form: find $\left(\mu_{\mathbb{S}}, \lambda_{\mathbb{S}}\right) \in$ $\Lambda_{\mathbb{S}} \times \Lambda_{\mathbb{S}}^{\prime}$ such that

$$
\underbrace{\left(\begin{array}{cc}
\mathcal{S}_{\Gamma_{\mathbb{S}}, \mathbb{S}} & \mathcal{I}_{\lambda} \\
\mathcal{S}_{\Gamma_{\mathbb{S}}, \mathbb{C}}-\mathcal{I}_{\lambda}
\end{array}\right)}_{\mathbb{S}_{D D}}\left(\begin{array}{c}
\mu_{\mathbb{S}} \\
\lambda_{\mathbb{S}}
\end{array}\right)=\left(\begin{array}{c}
g_{\Gamma_{\mathbb{S}}, \mathbb{S}} \\
g_{\Gamma_{\mathbb{S}}, \mathbb{C}}
\end{array}\right),
$$

where now $S_{D D}: \Lambda_{\mathbb{S}} \times \Lambda_{\mathbb{S}}^{\prime} \rightarrow \Lambda_{\mathbb{S}}^{\prime} \times \Lambda_{\mathbb{S}}^{\prime}$ is the block operator matrix associated to the interface problem in the two unknowns and $\mathcal{I}_{\lambda}$ is the identity operator in $\Lambda_{\mathbb{S}}^{\prime}$.

Remark 5 In the particular case of a 3D-1D coupling (see Sect. 2.3) we have that $\mathrm{S}_{D D}: \mathbb{R}^{2} \rightarrow \mathbb{R}^{2}$, indeed

$$
\mathrm{S}_{D D}=\left(\begin{array}{cc}
S_{1} & 1 \\
S_{3} & -1
\end{array}\right)
$$

so the exact representation of the operator is in fact a matrix $\mathrm{S}_{D D} \in \mathbb{R}^{2 \times 2}$. 
Proposition 3 There exists a unique pair $\left(\mu_{\mathbb{S}}, \lambda_{\mathbb{S}}\right) \in \Lambda_{\mathbb{S}} \times \Lambda_{\mathbb{S}}^{\prime}$ solution of (23). Moreover, there exists $C>0$ such that the solution satisfies

$$
\left\|\mu_{\mathbb{S}}\right\|_{\Lambda_{\mathbb{S}}}+\left\|\lambda_{\mathbb{S}}\right\|_{\Lambda_{\mathbb{S}}^{\prime}} \leq C\left(\left\|g_{\Gamma_{\mathbb{S}}, \mathbb{S}}\right\|_{\Lambda_{\mathbb{S}}^{\prime}}+\left\|g_{\Gamma_{\mathbb{S}}, \mathbb{C}}\right\|_{\Lambda_{\mathbb{S}}^{\prime}}\right) .
$$

Proof First of all note that the variational problem (23) can be written in compact form as follows: find $\theta_{\mathbb{S}} \in M$ such that

$$
r_{\Gamma_{\mathbb{S}}}\left(\theta_{\mathbb{S}}, \hat{\psi}_{\mathbb{S}}\right)=f_{\Gamma_{\mathbb{S}}}\left(\hat{\psi}_{\mathbb{S}}\right) \quad \forall \hat{\psi}_{\mathbb{S}} \in N
$$

where $M=\Lambda_{\mathbb{S}} \times \Lambda_{\mathbb{S}}^{\prime}, N=\Lambda_{\mathbb{S}} \times \Lambda_{\mathbb{S}}, \theta_{\mathbb{S}}=\left(\mu_{\mathbb{S}}, \lambda_{\mathbb{S}}\right)$ and $\hat{\psi}_{\mathbb{S}}=\left(\hat{\mu}_{\mathbb{S}}^{1}, \hat{\mu}_{\mathbb{S}}^{2}\right)$. Here we have that $r_{\Gamma_{\mathbb{S}}}: M \times N \rightarrow \mathbb{R}$ and $f_{\Gamma_{\mathbb{S}}}: N \rightarrow \mathbb{R}$. We also introduce the norms $\left\|\theta_{\mathbb{S}}\right\|_{M}=$ $\left\|\mu_{\mathbb{S}}\right\|_{\Lambda_{\mathbb{S}}}+\left\|\lambda_{\mathbb{S}}\right\|_{\Lambda_{\mathbb{S}}^{\prime}}$ and $\left\|\psi_{\mathbb{S}}\right\|_{N}=\left\|\mu_{\mathbb{S}}^{1}\right\|_{\Lambda_{\mathbb{S}}}+\left\|\mu_{\mathbb{S}}^{2}\right\|_{\Lambda_{\mathbb{S}}}$. Instead of the Lax-Milgram theorem as done in Proposition 2, here we apply the Neças theorem [17]. Bilinearity and continuity of $r_{\Gamma_{\mathbb{S}}}$ follow from the well-posedness of problems (7) and (9) and the same happens with the linearity and continuity of $f_{\Gamma_{\mathbb{S}}}$. The positivity in this problem holds if the following two conditions are satisfied: $r_{\Gamma_{\mathbb{S}}}$ is such that

$$
\sup _{\theta_{\mathbb{S}} \in M} r_{\Gamma_{\mathbb{S}}}\left(\theta_{\mathbb{S}}, \phi_{\mathbb{S}}\right)>0 \quad \forall \phi_{\mathbb{S}} \in N
$$

besides, there exists $\alpha>0$ such that

$$
\sup _{\phi_{\mathbb{S}} \in N} \frac{r_{\Gamma_{\mathbb{S}}}\left(\theta_{\mathbb{S}}, \phi_{\mathbb{S}}\right)}{\left\|\phi_{\mathbb{S}}\right\|_{N}} \geq \alpha\left\|\theta_{\mathbb{S}}\right\|_{M} \quad \forall \theta_{\mathbb{S}} \in M
$$

To show (26) let us take $\tilde{\theta}_{\mathbb{S}}=\left(\mu_{\mathbb{S}}^{2}, \lambda_{\mathbb{S}}^{1}\right)$ where $\lambda_{\mathbb{S}}^{1}$ can be characterized through the variational problem:

$$
a_{\mathbb{S}}\left(\mathcal{D}_{\mathbb{S}} \mu_{\mathbb{S}}^{1}, \hat{w}\right)=\left\langle\lambda_{\mathbb{S}}^{1}, \mathcal{R}_{\mathbb{S}} \hat{w}\right\rangle_{\mathbb{S}} \quad \forall \hat{w} \in \hat{U}_{\mathbb{S}}
$$

Then, for $\hat{w}=\mathcal{D}_{\mathbb{S}} \hat{\mu}_{\mathbb{S}}^{1}$ and for $\hat{w}=\mathcal{D}_{\mathbb{S}} \hat{\mu}_{\mathbb{S}}^{2}$ it is

$$
\begin{array}{ll}
a_{\mathbb{S}}\left(\mathcal{D}_{\mathbb{S}} \mu_{\mathbb{S}}^{1}, \mathcal{D}_{\mathbb{S}} \hat{\mu}_{\mathbb{S}}^{1}\right)=\left\langle\lambda_{\mathbb{S}}^{1}, \hat{\mu}_{\mathbb{S}}^{1}\right\rangle_{\mathbb{S}} & \forall \mathcal{D}_{\mathbb{S}} \hat{\mu}_{\mathbb{S}}^{1} \in \hat{U}_{\mathbb{S}}, \\
a_{\mathbb{S}}\left(\mathcal{D}_{\mathbb{S}} \mu_{\mathbb{S}}^{1}, \mathcal{D}_{\mathbb{S}} \hat{\mu}_{\mathbb{S}}^{2}\right)=\left\langle\lambda_{\mathbb{S}}^{1}, \hat{\mu}_{\mathbb{S}}^{2}\right\rangle_{\mathbb{S}} & \forall \mathcal{D}_{\mathbb{S}} \hat{\mu}_{\mathbb{S}}^{2} \in \hat{U}_{\mathbb{S}}
\end{array}
$$

With this choice, taking $\phi_{\mathbb{S}}=\left(\mu_{\mathbb{S}}^{1}, \mu_{\mathbb{S}}^{2}\right)$, and using the symmetry and coercivity of $s_{\Gamma_{\mathbb{S}}, \mathbb{S}}(\cdot, \cdot)$ and $s_{\Gamma_{\mathbb{S}}, \mathbb{C}}(\cdot, \cdot)$ we have

$$
\begin{aligned}
& r_{\Gamma_{\mathbb{S}}}\left(\tilde{\theta}_{\mathbb{S}}, \phi_{\mathbb{S}}\right)=s_{\Gamma_{\mathbb{S}}, \mathbb{S}}\left(\mu_{\mathbb{S}}^{2}, \mu_{\mathbb{S}}^{1}\right)+s_{\Gamma_{\mathbb{S}}, \mathbb{C}}\left(\mu_{\mathbb{S}}^{2}, \mu_{\mathbb{S}}^{2}\right)+\left\langle\lambda_{\mathbb{S}}^{1}, \mu_{\mathbb{S}}^{1}\right\rangle_{\mathbb{S}}-\left\langle\lambda_{\mathbb{S}}^{1}, \mu_{\mathbb{S}}^{2}\right\rangle_{\mathbb{S}} \\
& \quad=s_{\Gamma_{\mathbb{S}}, \mathbb{S}}\left(\mu_{\mathbb{S}}^{1}, \mu_{\mathbb{S}}^{1}\right)+s_{\Gamma_{\mathbb{S}}, \mathbb{C}}\left(\mu_{\mathbb{S}}^{2}, \mu_{\mathbb{S}}^{2}\right) \geq \alpha_{\mathbb{S}}\left\|\mu_{\mathbb{S}}^{1}\right\|_{\Lambda_{\mathbb{S}}}^{2}+\alpha_{\mathbb{C}}\left\|\mu_{\mathbb{S}}^{2}\right\|_{\Lambda_{\mathbb{S}}}^{2} \geq \alpha\left\|\phi_{\mathbb{S}}\right\|_{N}^{2} .
\end{aligned}
$$

Since this is valid for all $\phi_{\mathbb{S}} \in N$ and is valid for a particular $\tilde{\theta}_{\mathbb{S}} \in M$ we have that

$$
\sup _{\theta_{\mathbb{S}} \in M} r_{\Gamma_{\mathbb{S}}}\left(\theta_{\mathbb{S}}, \phi_{\mathbb{S}}\right) \geq r_{\Gamma_{\mathbb{S}}}\left(\tilde{\theta}_{\mathbb{S}}, \phi_{\mathbb{S}}\right) \geq \alpha\left\|\phi_{\mathbb{S}}\right\|_{N}^{2}>0 \quad \forall \phi_{\mathbb{S}} \in N,
$$


which is (26). To prove (27) we choose $\tilde{\phi}_{\mathbb{S}}=\left(\tilde{\mu}_{\mathbb{S}}, \frac{1}{2} \tilde{\mu}_{\mathbb{S}}\right)$, for which it is

$$
\begin{aligned}
r_{\Gamma_{\mathbb{S}}}\left(\theta_{\mathbb{S}}, \tilde{\phi}_{\mathbb{S}}\right) & =s_{\Gamma_{\mathbb{S}}, \mathbb{S}}\left(\mu_{\mathbb{S}}, \tilde{\mu}_{\mathbb{S}}\right)+\frac{1}{2} s_{\Gamma_{\mathbb{S}}, \mathbb{C}}\left(\mu_{\mathbb{S}}, \tilde{\mu}_{\mathbb{S}}\right)+\left\langle\lambda_{\mathbb{S}}, \tilde{\mu}_{\mathbb{S}}\right\rangle_{\mathbb{S}}-\frac{1}{2}\left\langle\lambda_{\mathbb{S}}, \tilde{\mu}_{\mathbb{S}}\right\rangle_{\mathbb{S}} \\
& =s_{\Gamma_{\mathbb{S}}, \mathbb{S}}\left(\mu_{\mathbb{S}}, \tilde{\mu}_{\mathbb{S}}\right)+\frac{1}{2} s_{\Gamma_{\mathbb{S}}, \mathbb{C}}\left(\mu_{\mathbb{S}}, \tilde{\mu}_{\mathbb{S}}\right)+\frac{1}{2}\left\langle\lambda_{\mathbb{S}}, \tilde{\mu}_{\mathbb{S}}\right\rangle_{\mathbb{S}}
\end{aligned}
$$

Dividing by $\left\|\tilde{\phi}_{\mathbb{S}}\right\|_{N}=\frac{3}{2}\left\|\tilde{\mu}_{\mathbb{S}}\right\|_{\Lambda_{\mathbb{S}}}$ we obtain

$$
\frac{r_{\Gamma_{\mathbb{S}}}\left(\theta_{\mathbb{S}}, \tilde{\phi}_{\mathbb{S}}\right)}{\left\|\tilde{\phi}_{\mathbb{S}}\right\|_{N}}=\frac{2}{3} \frac{s_{\Gamma_{\mathbb{S}}, \mathbb{S}}\left(\mu_{\mathbb{S}}, \tilde{\mu}_{\mathbb{S}}\right)}{\left\|\tilde{\mu}_{\mathbb{S}}\right\|_{\Lambda_{\mathbb{S}}}}+\frac{1}{3} \frac{s_{\Gamma_{\mathbb{S}}, \mathbb{C}}\left(\mu_{\mathbb{S}}, \tilde{\mu}_{\mathbb{S}}\right)}{\left\|\tilde{\mu}_{\mathbb{S}}\right\|_{\Lambda_{\mathbb{S}}}}+\frac{1}{3} \frac{\left\langle\lambda_{\mathbb{S}}, \tilde{\mu}_{\mathbb{S}}\right\rangle_{\mathbb{S}}}{\left\|\tilde{\mu}_{\mathbb{S}}\right\|_{\Lambda_{\mathbb{S}}}} .
$$

Notice that taking the supremum over $\tilde{\phi}_{\mathbb{S}} \in N$ implies taking the supremum over $\tilde{\mu}_{\mathbb{S}} \in \Lambda_{\mathbb{S}}$. In addition, the supremum over $\phi_{\mathbb{S}} \in N$ is bounded below by the supremum over $\tilde{\phi}_{\mathbb{S}} \in N$ (in the latter case we are restricting the supremum to all $\tilde{\phi}_{\mathbb{S}}$ with a very particular form equal to $\left.\left(\tilde{\mu}_{\mathbb{S}}, \frac{1}{2} \tilde{\mu}_{\mathbb{S}}\right)\right)$. Therefore

$$
\begin{aligned}
& \sup _{\phi_{\mathbb{S}} \in N} \frac{r_{\Gamma_{\mathbb{S}}}\left(\theta_{\mathbb{S}}, \phi_{\mathbb{S}}\right)}{\left\|\phi_{\mathbb{S}}\right\|_{N}} \geq \sup _{\tilde{\phi}_{\mathbb{S}} \in N} \frac{r_{\Gamma_{\mathbb{S}}}\left(\theta_{\mathbb{S}}, \tilde{\phi}_{\mathbb{S}}\right)}{\left\|\tilde{\phi}_{\mathbb{S}}\right\|_{N}} \\
& =\sup _{\tilde{\mu}_{\mathbb{S}} \in \Lambda_{\mathbb{S}}}\left[\frac{2}{3} \frac{s_{\Gamma_{\mathbb{S}}, \mathbb{S}}\left(\mu_{\mathbb{S}}, \tilde{\mu}_{\mathbb{S}}\right)}{\left\|\tilde{\mu}_{\mathbb{S}}\right\|_{\Lambda_{\mathbb{S}}}}+\frac{1}{3} \frac{s_{\Gamma_{\mathbb{S}}, \mathbb{C}}\left(\mu_{\mathbb{S}}, \tilde{\mu}_{\mathbb{S}}\right)}{\left\|\tilde{\mu}_{\mathbb{S}}\right\|_{\Lambda_{\mathbb{S}}}}+\frac{1}{3} \frac{\left\langle\lambda_{\mathbb{S}}, \tilde{\mu}_{\mathbb{S}}\right\rangle_{\mathbb{S}}}{\left\|\tilde{\mu}_{\mathbb{S}}\right\|_{\Lambda_{\mathbb{S}}}}\right] .
\end{aligned}
$$

Recalling that $s_{\Gamma_{\mathbb{S}}, \mathbb{S}}$ and $s_{\Gamma_{\mathbb{S}}, \mathbb{C}}$ are coercive, using the definition of the norm for $\Lambda_{\mathbb{S}}^{\prime}$ and noting that (28) is valid for all $\theta_{\mathbb{S}} \in M$, we get

$$
\sup _{\phi_{\mathbb{S}} \in N} \frac{r_{\Gamma_{\mathbb{S}}}\left(\theta_{\mathbb{S}}, \phi_{\mathbb{S}}\right)}{\left\|\phi_{\mathbb{S}}\right\|_{N}} \geq \frac{2}{3} \tilde{\alpha}_{\mathbb{S}}\left\|\mu_{\mathbb{S}}\right\|_{\Lambda_{\mathbb{S}}}+\frac{1}{3} \tilde{\alpha}_{\mathbb{C}}\left\|\mu_{\mathbb{S}}\right\|_{\Lambda_{\mathbb{S}}}+\frac{1}{3}\left\|\lambda_{\mathbb{S}}\right\|_{\Lambda_{\mathbb{S}}^{\prime}} \geq \alpha\left\|\theta_{\mathbb{S}}\right\|_{M},
$$

from which (27) follows. Hence, the existence and uniqueness of the solution $\left(\mu_{\mathbb{S}}\right.$, $\left.\lambda_{\mathbb{S}}\right) \in \Lambda_{\mathbb{S}} \times \Lambda_{\mathbb{S}}^{\prime}$ (that is $\theta_{\mathbb{S}} \in M$ ) is ensured by the Neças theorem. The estimate (25) is also a corollary of the Neças theorem.

\subsubsection{Approach 2: Dirichlet-and-Neumann decomposition}

In this approach we will slightly change the way we split $u_{\mathbb{S}}$ and $u_{\mathbb{C}}$. More precisely, for the $\mathbb{S D}$-model we consider a Dirichlet problem and for the $\mathbb{C D}$-model a Neumann problem. We therefore set

$$
u_{\mathbb{S}}=u_{\mathbb{S}}^{I}+\mathcal{D}_{\mathbb{S}} \mu_{\mathbb{S}}, \quad u_{\mathbb{C}}=u_{\mathbb{C}}^{J}+\mathcal{N}_{\mathbb{C}} \lambda_{\mathbb{S}}
$$

where operators $\mathcal{D}_{\mathbb{S}}$ and $\mathcal{N}_{\mathbb{C}}$ are defined according to (7) and (11). In turn, $u_{\mathbb{C}}^{J} \in U_{\mathbb{C}}$ is given by the solution of the following variational problem:

$$
a_{\mathbb{C}}\left(u_{\mathbb{C}}^{J}, \hat{u}_{\mathbb{C}}^{J}\right)=f_{\mathbb{C}}\left(\hat{u}_{\mathbb{C}}^{J}\right) \quad \forall \hat{u}_{\mathbb{C}}^{J} \in \hat{U}_{\mathbb{C}}
$$


The denomination Dirichlet-and-Neumann decomposition stems from the fact that the splitting (29) involves Dirichlet and Neumann sub-problems, respectively.

The admissible variations in this case are

$$
\hat{u}_{\mathbb{S}}=\hat{u}_{\mathbb{S}}^{I}+\mathcal{D}_{\mathbb{S}} \hat{\mu}_{\mathbb{S}}, \quad \hat{u}_{\mathbb{C}}=\hat{u}_{\mathbb{C}}^{J}+\mathcal{N}_{\mathbb{C}} \hat{\lambda}_{\mathbb{S}}
$$

Then, our variational problem becomes: given $u_{\mathbb{S}}^{I}$ and $u_{\mathbb{C}}^{J}$ solutions of (16) and (30), find $\left(\mu_{\mathbb{S}}, \lambda_{\mathbb{S}}\right) \in \Lambda_{\mathbb{S}} \times \Lambda_{\mathbb{S}}^{\prime}$ such that

$$
\begin{aligned}
a_{\mathbb{S}} & \left(u_{\mathbb{S}}^{I}+\mathcal{D}_{\mathbb{S}} \mu_{\mathbb{S}}, \hat{u}_{\mathbb{S}}^{I}+\mathcal{D}_{\mathbb{S}} \hat{\mu}_{\mathbb{S}}\right)+a_{\mathbb{C}}\left(u_{\mathbb{C}}^{J}+\mathcal{N}_{\mathbb{C}} \lambda_{\mathbb{S}}, \hat{u}_{\mathbb{C}}^{J}+\mathcal{N}_{\mathbb{C}} \hat{\lambda}_{\mathbb{S}}\right) \\
& +\left\langle\lambda_{\mathbb{S}}, \hat{u}_{\mathbb{S}}^{I}-\mathcal{R}_{\mathbb{S}} \hat{u}_{\mathbb{C}}^{J}\right\rangle_{\mathbb{S}}+\left\langle\lambda_{\mathbb{S}}, \hat{\mu}_{\mathbb{S}}-\mathcal{R}_{\mathbb{S}}\left(\mathcal{N}_{\mathbb{C}} \hat{\lambda}_{\mathbb{S}}\right)\right\rangle_{\mathbb{S}} \\
& +\left\langle\hat{\lambda}_{\mathbb{S}}, u_{\mathbb{S}}^{I}-\mathcal{R}_{\mathbb{S}} u_{\mathbb{C}}^{J}\right\rangle_{\mathbb{S}}+\left\langle\hat{\lambda}_{\mathbb{S}}, \mu_{\mathbb{S}}-\mathcal{R}_{\mathbb{S}}\left(\mathcal{N}_{\mathbb{C}} \lambda_{\mathbb{S}}\right)\right\rangle_{\mathbb{S}} \\
= & f_{\mathbb{S}}\left(\hat{u}_{\mathbb{S}}^{I}+\mathcal{D}_{\mathbb{S}} \hat{\mu}_{\mathbb{S}}\right)+f_{\mathbb{C}}\left(\hat{u}_{\mathbb{C}}^{J}+\mathcal{N}_{\mathbb{C}} \hat{\lambda}_{\mathbb{S}}\right) \quad \forall\left(\hat{\mu}_{\mathbb{S}}, \hat{\lambda}_{\mathbb{S}}\right) \in \Lambda_{\mathbb{S}} \times \Lambda_{\mathbb{S}}^{\prime}
\end{aligned}
$$

Rearranging terms, using (7) and (16) as in (18), and using (11) and (30), and the fact that $u_{\mathbb{S}}^{I} \in U_{\mathbb{S}}^{0}$ we obtain the problem:

$$
\begin{aligned}
a_{\mathbb{S}} & \left(\mathcal{D}_{\mathbb{S}} \mu_{\mathbb{S}}, \mathcal{D}_{\mathbb{S}} \hat{\mu}_{\mathbb{S}}\right)+a_{\mathbb{C}}\left(\mathcal{N}_{\mathbb{C}} \lambda_{\mathbb{S}}, \mathcal{N}_{\mathbb{C}} \hat{\lambda}_{\mathbb{S}}\right) \\
& +\left\langle\lambda_{\mathbb{S}}, \hat{\mu}_{\mathbb{S}}-\mathcal{R}_{\mathbb{S}}\left(\mathcal{N}_{\mathbb{C}} \hat{\lambda}_{\mathbb{S}}\right)\right\rangle_{\mathbb{S}}+\left\langle\hat{\lambda}_{\mathbb{S}}, \mu_{\mathbb{S}}-\mathcal{R}_{\mathbb{S}}\left(u_{\mathbb{C}}^{J}+\mathcal{N}_{\mathbb{C}} \lambda_{\mathbb{S}}\right)\right\rangle_{\mathbb{S}} \\
= & f_{\mathbb{S}}\left(\mathcal{D}_{\mathbb{S}} \hat{\mu}_{\mathbb{S}}\right)-a_{\mathbb{S}}\left(u_{\mathbb{S}}^{I}, \mathcal{D}_{\mathbb{S}} \hat{\mu}_{\mathbb{S}}\right) \\
& +f_{\mathbb{C}}\left(\mathcal{N}_{\mathbb{C}} \hat{\lambda}_{\mathbb{S}}\right)-a_{\mathbb{C}}\left(u_{\mathbb{C}}^{J}, \mathcal{N}_{\mathbb{C}} \hat{\lambda}_{\mathbb{S}}\right) \quad \forall\left(\hat{\mu}_{\mathbb{S}}, \hat{\lambda}_{\mathbb{S}}\right) \in \Lambda_{\mathbb{S}} \times \Lambda_{\mathbb{S}}^{\prime} .
\end{aligned}
$$

Now, notice that, due to (30) and making use of (11) we obtain

$$
\begin{gathered}
a_{\mathbb{S}}\left(\mathcal{D}_{\mathbb{S}} \mu_{\mathbb{S}}, \mathcal{D}_{\mathbb{S}} \hat{\mu}_{\mathbb{S}}\right)+\left\langle\lambda_{\mathbb{S}}, \hat{\mu}_{\mathbb{S}}\right\rangle_{\mathbb{S}}+\left\langle\hat{\lambda}_{\mathbb{S}}, \mu_{\mathbb{S}}-\mathcal{R}_{\mathbb{S}}\left(u_{\mathbb{C}}^{J}+\mathcal{N}_{\mathbb{C}} \lambda_{\mathbb{S}}\right)\right\rangle_{\mathbb{S}} \\
=f_{\mathbb{S}}\left(\mathcal{D}_{\mathbb{S}} \hat{\mu}_{\mathbb{S}}\right)-a_{\mathbb{S}}\left(u_{\mathbb{S}}^{I}, \mathcal{D}_{\mathbb{S}} \hat{\mu}_{\mathbb{S}}\right) \quad \forall\left(\hat{\mu}_{\mathbb{S}}, \hat{\lambda}_{\mathbb{S}}\right) \in \Lambda_{\mathbb{S}} \times \Lambda_{\mathbb{S}}^{\prime} .
\end{gathered}
$$

Once again, here we keep both variables, $\mu_{\mathbb{S}}$ and $\lambda_{\mathbb{S}}$, for which the two equations are provided by $\hat{\mu}_{\mathbb{S}}$ and $\hat{\lambda}_{\mathbb{S}}$. In this case, the problem is expressed in compact form, with obvious meaning of notation, as follows: find $\left(\mu_{\mathbb{S}}, \lambda_{\mathbb{S}}\right) \in \Lambda_{\mathbb{S}} \times \Lambda_{\mathbb{S}}^{\prime}$ such that

$$
\begin{array}{cc}
s_{\Gamma_{\mathbb{S}}, \mathbb{S}}\left(\mu_{\mathbb{S}}, \hat{\mu}_{\mathbb{S}}\right)+\left\langle\lambda_{\mathbb{S}}, \hat{\mu}_{\mathbb{S}}\right\rangle_{\mathbb{S}}=g_{\Gamma_{\mathbb{S}}, \mathbb{S}}\left(\hat{\mu}_{\mathbb{S}}\right) \quad \forall \hat{\mu}_{\mathbb{S}} \in \Lambda_{\mathbb{S}} \\
\left\langle\hat{\lambda}_{\mathbb{S}}, \mu_{\mathbb{S}}\right\rangle_{\mathbb{S}}-\left\langle\hat{\lambda}_{\mathbb{S}}, \mathcal{R}_{\mathbb{S}}\left(\mathcal{N}_{\mathbb{C}} \lambda_{\mathbb{S}}\right)\right\rangle_{\mathbb{S}}=\left\langle\hat{\lambda}_{\mathbb{S}}, \mathcal{R}_{\mathbb{S}} u_{\mathbb{C}}^{J}\right\rangle_{\mathbb{S}} \quad \forall \hat{\lambda}_{\mathbb{S}} \in \Lambda_{\mathbb{S}}^{\prime} .
\end{array}
$$

Similarly to (24) we can write (31) in block operator matrix form

$$
\underbrace{\left(\begin{array}{cc}
\mathcal{S}_{\Gamma_{\mathbb{S}}, \mathbb{S}} & \mathcal{I}_{\lambda} \\
\mathcal{I}_{\mu} & -\mathcal{T}_{\Gamma_{\mathbb{S}}, \mathbb{C}}
\end{array}\right)}_{\mathbb{S}_{D N}}\left(\begin{array}{c}
\mu_{\mathbb{S}} \\
\lambda_{\mathbb{S}}
\end{array}\right)=\left(\begin{array}{c}
g_{\Gamma_{\mathbb{S}}, \mathbb{S}} \\
\mathcal{R}_{\mathbb{S}} u_{\mathbb{C}}^{J}
\end{array}\right)
$$


where in this situation the definition of the operator $\mathcal{T}_{\Gamma_{\mathbb{S}}, \mathbb{C}}$ is $\left\langle\hat{\lambda}_{\mathbb{S}}, \mathcal{T}_{\Gamma_{\mathbb{S}}, \mathbb{C}} \lambda_{\mathbb{S}}\right\rangle_{\mathbb{S}}=$ $\left\langle\hat{\lambda}_{\mathbb{S}}, \mathcal{R}_{\mathbb{S}}\left(\mathcal{N}_{\mathbb{C}} \lambda_{\mathbb{S}}\right)\right\rangle_{\mathbb{S}}$ and it is $S_{D N}: \Lambda_{\mathbb{S}} \times \Lambda_{\mathbb{S}}^{\prime} \rightarrow \Lambda_{\mathbb{S}}^{\prime} \times \Lambda_{\mathbb{S}}$. Here $\mathcal{I}_{\lambda}$ and $\mathcal{I}_{\mu}$ are the identity operators in $\Lambda_{\mathbb{S}}^{\prime}$ and $\Lambda_{\mathbb{S}}$, respectively.

Proposition 4 There exists a unique pair $\left(\mu_{\mathbb{S}}, \lambda_{\mathbb{S}}\right) \in \Lambda_{\mathbb{S}} \times \Lambda_{\mathbb{S}}^{\prime}$ solution of (31), moreover there exists $C>0$ such that

$$
\left\|\mu_{\mathbb{S}}\right\|_{\Lambda_{\mathbb{S}}}+\left\|\lambda_{\mathbb{S}}\right\|_{\Lambda_{\mathbb{S}}^{\prime}} \leq C\left(\left\|g_{\Gamma_{\mathbb{S}}, \mathbb{S}}\right\|_{\Lambda_{\mathbb{S}}^{\prime}}+\left\|\mathcal{R}_{\mathbb{S}} u_{\mathbb{C}}^{J}\right\|_{\Lambda_{\mathbb{S}}}\right)
$$

Proof It follows similar guidelines to those employed in Proposition 3 and is not presented here for the sake of brevity.

\subsubsection{Approach 3: Neumann-and-Neumann decomposition}

Another possible decomposition involves the solution of Neumann problems for both the $\mathbb{C D}$-model and the $\mathbb{S D}$-model. The decompositions of the solution functions in this case are as follows

$$
u_{\mathbb{S}}=u_{\mathbb{S}}^{J}+\mathcal{N}_{\mathbb{S}} \lambda_{\mathbb{S}}, \quad u_{\mathbb{C}}=u_{\mathbb{C}}^{J}+\mathcal{N}_{\mathbb{C}} \lambda_{\mathbb{S}}
$$

with admissible variations given by

$$
\hat{u}_{\mathbb{S}}=\hat{u}_{\mathbb{S}}^{J}+\mathcal{N}_{\mathbb{S}} \hat{\lambda}_{\mathbb{S}}^{1}, \quad \hat{u}_{\mathbb{C}}=\hat{u}_{\mathbb{C}}^{J}+\mathcal{N}_{\mathbb{C}} \hat{\lambda}_{\mathbb{S}}^{2}
$$

Proceeding as before we obtain the following interface formulation: find $\left(\mu_{\mathbb{S}}, \lambda_{\mathbb{S}}\right) \in$ $\Lambda_{\mathbb{S}} \times \Lambda_{\mathbb{S}}^{\prime}$ such that

$$
\begin{gathered}
\left\langle\hat{\lambda}_{\mathbb{S}}^{1}, \mu_{\mathbb{S}}\right\rangle_{\mathbb{S}}-\left\langle\hat{\lambda}_{\mathbb{S}}^{1}, \mathcal{N}_{\mathbb{S}} \lambda_{\mathbb{S}}\right\rangle_{\mathbb{S}}=\left\langle\hat{\lambda}_{\mathbb{S}}^{1}, u_{\mathbb{S}}^{J}\right\rangle_{\mathbb{S}} \quad \forall \hat{\lambda}_{\mathbb{S}}^{1} \in \Lambda_{\mathbb{S}}^{\prime}, \\
\left\langle\hat{\lambda}_{\mathbb{S}}^{2}, \mu_{\mathbb{S}}\right\rangle_{\mathbb{S}}-\left\langle\hat{\lambda}_{\mathbb{S}}^{2}, \mathcal{R}_{\mathbb{S}}\left(\mathcal{N}_{\mathbb{C}} \lambda_{\mathbb{S}}\right)\right\rangle_{\mathbb{S}}=\left\langle\hat{\lambda}_{\mathbb{S}}^{2}, \mathcal{R}_{\mathbb{S}} u_{\mathbb{C}}^{J}\right\rangle_{\mathbb{S}} \quad \forall \hat{\lambda}_{\mathbb{S}}^{2} \in \Lambda_{\mathbb{S}}^{\prime} .
\end{gathered}
$$

In block operator matrix form (33) corresponds to

$$
\underbrace{\left(\begin{array}{l}
\mathcal{I}_{\mu}-\mathcal{T}_{\Gamma_{\mathbb{S}}, \mathbb{S}} \\
\mathcal{I}_{\mu}-\mathcal{T}_{\Gamma_{\mathbb{S}}, \mathbb{C}}
\end{array}\right)}_{\mathrm{S}_{N N}}\left(\begin{array}{c}
\mu_{\mathbb{S}} \\
\lambda_{\mathbb{S}}
\end{array}\right)=\left(\begin{array}{c}
u_{\mathbb{S}}^{J} \\
\mathcal{R}_{\mathbb{S}} u_{\mathbb{C}}^{J}
\end{array}\right)
$$

where, as before, the operator $\mathcal{T}_{\Gamma_{\mathbb{S}}, \mathbb{S}}$ is defined by $\left\langle\hat{\lambda}_{\mathbb{S}}^{1}, \mathcal{T}_{\Gamma_{\mathbb{S}}, \mathbb{S}} \lambda_{\mathbb{S}}\right\rangle_{\mathbb{S}}=\left\langle\hat{\lambda}_{\mathbb{S}}^{1}, \mathcal{N}_{\mathbb{S}} \lambda_{\mathbb{S}}\right\rangle_{\mathbb{S}}$, and then it is $S_{N N}: \Lambda_{\mathbb{S}} \times \Lambda_{\mathbb{S}}^{\prime} \rightarrow \Lambda_{\mathbb{S}} \times \Lambda_{\mathbb{S}}$.

Proposition 5 There exists a unique pair $\left(\mu_{\mathbb{S}}, \lambda_{\mathbb{S}}\right) \in \Lambda_{\mathbb{S}} \times \Lambda_{\mathbb{S}}^{\prime}$ solution of (33), and a constant $C>0$ such that

$$
\left\|\mu_{\mathbb{S}}\right\|_{\Lambda_{\mathbb{S}}}+\left\|\lambda_{\mathbb{S}}\right\|_{\Lambda_{\mathbb{S}}^{\prime}} \leq C\left(\left\|u_{\mathbb{S}}^{J}\right\|_{\Lambda_{\mathbb{S}}}+\left\|\mathcal{R}_{\mathbb{S}} u_{\mathbb{C}}^{J}\right\|_{\Lambda_{\mathbb{S}}}\right)
$$




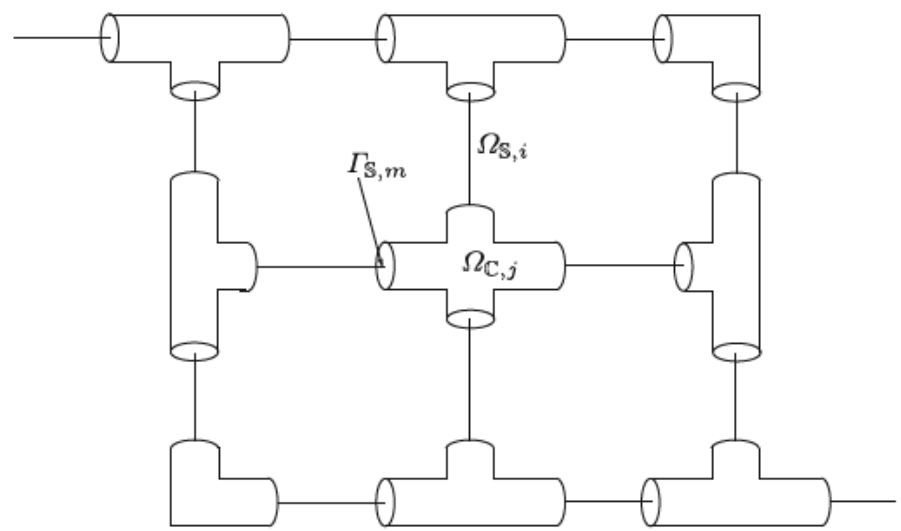

Fig. 4 Large system featuring several $\mathbb{C D}$ and $\mathrm{SD}$ sub-systems $\left(N_{\mathbb{C}}=9, N_{\mathbb{S}}=14, N=23, M=26\right)$. For simplicity in this figure we stick to the case $\mathbb{C}=3$ and $\mathbb{S}=1$

Proof The proof is analogous to the proofs of Propositions 3 and 4, and is omitted here for the sake of brevity.

Remark 6 The Dirichlet-and-Dirichlet, Dirichlet-and-Neumann, and Neumann-andNeumann approaches represent three equivalent forms of reformulating the same problem. Therefore, the (continuous) systems (24), (32) and (34) feature the same solution. Finally, notice that, if for modeling reasons other type of coupling conditions (e.g., of Robin type) have to be considered on the interface, they can be easily accommodated within the present framework.

\section{Analysis of multi-component systems}

In this section we extend the previous theory to the more general case of networks containing an arbitrary number of components. Then we study the specific problem involving the coupling of $\mathbb{C D}-\mathbb{S D}$ models $(\mathbb{S}=0,1)$ and explore some peculiarities arising in that case.

\subsection{Interface problems for multi-component systems}

Let us consider a network composed by $N$ components, $N_{\mathbb{C}}$ made by $\mathbb{C D}$-models and $N_{\mathbb{S}}$ by SD-models, as shown schematically in Fig. 4 . In this system we have $M$ coupling points, for each of them we identify the coupling interfaces $\Gamma_{\mathbb{S}, m}, m=1, \ldots, M$, to which we associate two unknowns in the present scalar problem, namely $\mu_{\mathbb{S}, m}$ and $\lambda_{\mathbb{S}, m}$.

Recall that the dimensionally-heterogeneous system seen in Fig. 4 is somehow a geometrical multi-scale representation of a dimensionally-homogeneous one. The variational formulation reads:

find $\left(\left\{u_{\mathbb{C}, j}\right\}_{j=1}^{N_{\mathbb{C}}},\left\{u_{\mathbb{S}, i}\right\}_{i=1}^{N_{\mathbb{S}}},\left\{\lambda_{\mathbb{S}, m}\right\}_{m=1}^{M}\right) \in \prod_{j=1}^{N_{\mathbb{C}}} U_{\mathbb{C}, j} \times \prod_{i=1}^{N_{\mathbb{S}}} U_{\mathbb{S}, i} \times \prod_{m=1}^{M} \Lambda_{\mathbb{S}, m}^{\prime}$ such that 


$$
\begin{gathered}
\sum_{j=1}^{N_{\mathbb{C}}} a_{\mathbb{C}, j}\left(u_{\mathbb{C}, j}, \hat{u}_{\mathbb{C}, j}\right)+\sum_{i=1}^{N_{\mathbb{S}}} a_{\mathbb{S}, i}\left(u_{\mathbb{S}, i}, \hat{u}_{\mathbb{S}, i}\right)+\sum_{m=1}^{M}\left\langle\lambda_{\mathbb{S}, m}, \hat{u}_{\mathbb{S}, i \mid m}-\mathcal{R}_{\mathbb{S}, m} \hat{u}_{\mathbb{C}, j \mid m}\right\rangle_{\mathbb{S}, m} \\
+\sum_{m=1}^{M}\left\langle\hat{\lambda}_{\mathbb{S}, m}, u_{\mathbb{S}, i \mid m}-\mathcal{R}_{\mathbb{S}, m} u_{\mathbb{C}, j \mid m}\right\rangle_{\mathbb{S}, m}=\sum_{i=1}^{N_{\mathbb{S}}} f_{\mathbb{S}, i}\left(\hat{u}_{\mathbb{S}, i}\right)+\sum_{j=1}^{N_{\mathbb{C}}} f_{\mathbb{C}, j}\left(\hat{u}_{\mathbb{C}, j}\right) \\
\forall\left(\left\{\hat{u}_{\mathbb{C}, j}\right\}_{j=1}^{N_{\mathbb{C}}},\left\{\hat{u}_{\mathbb{S}, i}\right\}_{i=1}^{N_{\mathbb{S}}},\left\{\hat{\lambda}_{\mathbb{S}, m}\right\}_{m=1}^{M}\right) \in \prod_{j=1}^{N_{\mathbb{C}}} U_{\mathbb{C}, j} \times \prod_{i=1}^{N_{\mathbb{S}}} U_{\mathbb{S}, i} \times \prod_{m=1}^{M} \Lambda_{\mathbb{S}, m}^{\prime} .
\end{gathered}
$$

The notation $u_{\mathbb{S}, i \mid m}$ is used to denote the restriction of $u_{\mathbb{S}, i}$ to the $m$-th interface $\Gamma_{\mathbb{S}, m}$ and so on.

To derive the interface formulation, and for the sake of simplicity, we will consider the situation in which we decompose the solution in each sub-model imposing a Dirichlet boundary condition, that is, imposing the value of $\mathcal{R}_{\mathbb{S}} u_{\mathbb{C}, j \mid m}$ over each interface $\Gamma_{\mathbb{C}, m}$. In this general setting one component can have more than one coupling interface. So, considering the $K_{j}$ and the $K_{i}$ coupling interfaces of the $j$-th complex and $i$-th simple components, respectively, the decompositions and the variations become

$$
\begin{aligned}
& u_{\mathbb{S}, i}=u_{\mathbb{S}, i}^{I}+\sum_{k=1}^{K_{i}} \mathcal{D}_{\mathbb{S}, i} R_{\mathbb{S}, i \mid k} \mu_{\mathbb{S}, m}, \quad \hat{u}_{\mathbb{S}, i}=\hat{u}_{\mathbb{S}, i}^{I}+\sum_{k=1}^{K_{i}} \mathcal{D}_{\mathbb{S}, i} R_{\mathbb{S}, i \mid k} \hat{\mu}_{\mathbb{S}, m}^{1}, \\
& u_{\mathbb{C}, j}=u_{\mathbb{C}, j}^{I}+\sum_{k=1}^{K_{j}} \mathcal{D}_{\mathbb{C}, j} R_{\mathbb{C}, j \mid k} \mu_{\mathbb{S}, m}, \quad \hat{u}_{\mathbb{C}, j}=\hat{u}_{\mathbb{C}, j}^{I}+\sum_{k=1}^{K_{j}} \mathcal{D}_{\mathbb{C}, j} R_{\mathbb{C}, j \mid k} \hat{\mu}_{\mathbb{S}, m}^{2},
\end{aligned}
$$

where now $\mathcal{D}_{\mathbb{C}, j}$ and $\mathcal{D}_{\mathbb{S}, i}$ are the extension operators defined by (7) and (9) in the corresponding components, and $u_{\mathbb{C}, j}^{I}$, and $u_{\mathbb{S}, i}^{I}$ are also the solutions of problems similar to those in (16). Moreover, the matrices $R_{\mathbb{C}, j \mid k} \in \mathbb{R}^{K_{j} \times M}$ and $R_{\mathbb{S}, i \mid k} \in \mathbb{R}^{K_{i} \times M}$ select among the interface unknowns $\mu_{\mathbb{S}, m}$ those associated to the $K_{j}$ or $K_{i}$ interfaces of the $j$-th or $i$-th component, respectively. Following similar steps to those which led us to Eq. (23) yields in this case: given the functions $\left\{u_{\mathbb{C}, j}^{I}\right\}_{j=1}^{N_{\mathbb{C}}}$ and $\left\{u_{\mathbb{S}, i}^{I}\right\}_{i=1}^{N_{\mathbb{S}}}$, find $\left(\left\{\mu_{\mathbb{S}, m}\right\}_{m=1}^{M},\left\{\lambda_{\mathbb{S}, m}\right\}_{m=1}^{M}\right) \in \prod_{m=1}^{M} \Lambda_{\mathbb{S}, m} \times \prod_{m=1}^{M} \Lambda_{\mathbb{S}, m}^{\prime}$ such that

$$
\begin{aligned}
\sum_{j=1}^{N_{\mathbb{C}}} & \sum_{k=1}^{K_{j}} a_{\mathbb{C}, j}\left(\mathcal{D}_{\mathbb{C}, j} R_{\mathbb{C}, j \mid k} \mu_{\mathbb{S}, m}, \mathcal{D}_{\mathbb{C}, j} R_{\mathbb{C}, j \mid k} \hat{\mu}_{\mathbb{S}, m}^{2}\right) \\
& +\sum_{i=1}^{N_{\mathbb{S}}} \sum_{k=1}^{K_{i}} a_{\mathbb{S}, i}\left(\mathcal{D}_{\mathbb{S}, i} R_{\mathbb{S}, i \mid k} \mu_{\mathbb{S}, m}, \mathcal{D}_{\mathbb{S}, i} R_{\mathbb{S}, i \mid k} \hat{\mu}_{\mathbb{S}, m}^{1}\right)+\sum_{m=1}^{M}\left\langle\lambda_{\mathbb{S}, m}, \hat{\mu}_{\mathbb{S}, m}^{1}-\hat{\mu}_{\mathbb{S}, m}^{2}\right\rangle_{\mathbb{S}, m} \\
& =\sum_{i=1}^{N_{\mathbb{S}}} \sum_{k=1}^{K_{i}}\left(f_{\mathbb{S}, i}\left(\mathcal{D}_{\mathbb{S}, i} R_{\mathbb{S}, i \mid k} \hat{\mu}_{\mathbb{S}, m}^{1}\right)-a_{\mathbb{S}, i}\left(u_{\mathbb{S}, i}^{I}, \mathcal{D}_{\mathbb{S}, i} R_{\mathbb{S}, i \mid k} \hat{\mu}_{\mathbb{S}, m}^{1}\right)\right)
\end{aligned}
$$




$$
\begin{aligned}
& +\sum_{j=1}^{N_{\mathbb{C}}} \sum_{k=1}^{K_{j}}\left(f_{\mathbb{C}, j}\left(\mathcal{D}_{\mathbb{C}, j} R_{\mathbb{C}, j \mid k} \hat{\mu}_{\mathbb{S}, m}^{2}\right)-a_{\mathbb{C}, j}\left(u_{\mathbb{C}, j}^{I}, \mathcal{D}_{\mathbb{C}, j} R_{\mathbb{C}, j \mid k} \hat{\mu}_{\mathbb{S}, m}^{2}\right)\right) \\
& \forall\left(\left\{\hat{\mu}_{\mathbb{S}, m}^{1}\right\}_{m=1}^{M},\left\{\hat{\mu}_{\mathbb{S}, m}^{2}\right\}_{m=1}^{M}\right) \in \prod_{m=1}^{M} \Lambda_{\mathbb{S}, m} \times \prod_{m=1}^{M} \Lambda_{\mathbb{S}, m} .
\end{aligned}
$$

In compact form, with obvious meaning of notation, the interface variational problem is written as follows: given $\boldsymbol{u}_{\mathbb{S}}^{I}$ and $\boldsymbol{u}_{\mathbb{C}}^{I}$, find $\left(\boldsymbol{\mu}_{\mathbb{S}}, \lambda_{\mathbb{S}}\right) \in \boldsymbol{\Lambda}_{\mathbb{S}} \times \boldsymbol{\Lambda}_{\mathbb{S}}^{\prime}$ such that

$$
\begin{array}{ll}
s_{\Gamma_{\mathbb{S}}, \mathbb{S}}\left(\boldsymbol{\mu}_{\mathbb{S}}, \hat{\boldsymbol{\mu}}_{\mathbb{S}}^{1}\right)+\left\langle\lambda_{\mathbb{S}}, \hat{\boldsymbol{\mu}}_{\mathbb{S}}^{1}\right\rangle_{\mathbb{S}}=\boldsymbol{g}_{\Gamma_{\mathbb{S}}, \mathbb{S}}\left(\hat{\boldsymbol{\mu}}_{\mathbb{S}}^{1}\right) & \forall \hat{\boldsymbol{\mu}}_{\mathbb{S}}^{1} \in \boldsymbol{\Lambda}_{\mathbb{S}}, \\
s_{\Gamma_{\mathbb{S}}, \mathbb{C}}\left(\boldsymbol{\mu}_{\mathbb{S}}, \hat{\boldsymbol{\mu}}_{\mathbb{S}}^{2}\right)-\left\langle\lambda_{\mathbb{S}}, \hat{\boldsymbol{\mu}}_{\mathbb{S}}^{2}\right\rangle_{\mathbb{S}}=\boldsymbol{g}_{\Gamma_{\mathbb{S}}, \mathbb{C}}\left(\hat{\boldsymbol{\mu}}_{\mathbb{S}}^{2}\right) & \forall \hat{\boldsymbol{\mu}}_{\mathbb{S}}^{2} \in \boldsymbol{\Lambda}_{\mathbb{S}} .
\end{array}
$$

We have therefore the following formulation, which is a counterpart of (24):

$$
\left(\begin{array}{cc}
\mathcal{S}_{\Gamma_{\mathbb{S}}, \mathbb{S}} & \mathcal{I}_{\lambda} \\
\mathcal{S}_{\Gamma_{\mathbb{S}}, \mathbb{C}} & -\mathcal{I}_{\lambda}
\end{array}\right)\left(\begin{array}{l}
\boldsymbol{\mu}_{\mathbb{S}} \\
\lambda_{\mathbb{S}}
\end{array}\right)=\left(\begin{array}{l}
\boldsymbol{g}_{\Gamma_{\mathbb{S}}, \mathbb{S}} \\
\boldsymbol{g}_{\Gamma_{\mathbb{S}}, \mathbb{C}}
\end{array}\right) .
$$

We can state the following result (proof as in Proposition 3).

Proposition 6 There exists a unique pair $\left(\boldsymbol{\mu}_{\mathbb{S}}, \lambda_{\mathbb{S}}\right) \in \Lambda_{\mathbb{S}} \times \Lambda_{\mathbb{S}}^{\prime}$ solution of (35) and a constant $C>0$ such that

$$
\left\|\boldsymbol{\mu}_{\mathbb{S}}\right\|_{\boldsymbol{\Lambda}_{\mathbb{S}}}+\left\|\lambda_{\mathbb{S}}\right\|_{\boldsymbol{\Lambda}_{\mathbb{S}}^{\prime}} \leq C\left(\left\|\boldsymbol{g}_{\Gamma_{\mathbb{S}}, \mathbb{S}}\right\|_{\boldsymbol{\Lambda}_{\mathbb{S}}^{\prime}}+\left\|\boldsymbol{g}_{\Gamma_{\mathbb{S}}, \mathbb{C}}\right\|_{\Lambda_{\mathbb{S}}^{\prime}}\right) .
$$

The Steklov-Poincare formulation is obtained by adding the two equations in (35) and assuming that $\hat{\boldsymbol{\mu}}_{\mathbb{S}}^{1}=\hat{\boldsymbol{\mu}}_{\mathbb{S}}^{2}=\hat{\boldsymbol{\mu}}_{\mathbb{S}}$, leading to

$$
s_{\Gamma_{\mathbb{S}}, \mathbb{S}}\left(\boldsymbol{\mu}_{\mathbb{S}}, \hat{\boldsymbol{\mu}}_{\mathbb{S}}\right)+s_{\Gamma_{\mathbb{S}}, \mathbb{C}}\left(\boldsymbol{\mu}_{\mathbb{S}}, \hat{\boldsymbol{\mu}}_{\mathbb{S}}\right)=\boldsymbol{g}_{\Gamma_{\mathbb{S}}, \mathbb{S}}\left(\hat{\boldsymbol{\mu}}_{\mathbb{S}}\right)+\boldsymbol{g}_{\Gamma_{\mathbb{S}}, \mathbb{C}}\left(\hat{\boldsymbol{\mu}}_{\mathbb{S}}\right) \quad \forall \hat{\boldsymbol{\mu}}_{\mathbb{S}} \in \boldsymbol{\Lambda}_{\mathbb{S}}
$$

Equivalently, we can write

$$
s_{\Gamma_{\mathbb{S}}}\left(\boldsymbol{\mu}_{\mathbb{S}}, \hat{\mu}_{\mathbb{S}}\right)=g_{\Gamma_{\mathbb{S}}}\left(\hat{\mu}_{\mathbb{S}}\right) \quad \forall \hat{\mu}_{\mathbb{S}} \in \boldsymbol{\Lambda}_{\mathbb{S}}
$$

The existence and uniqueness of a solution to problem (37) can be proved in the same manner as in Proposition 2.

\subsection{Coupling $\mathbb{C D}$ - $\mathbb{S D}$ models $(\mathbb{S}=0,1)$ in the multi-component case}

From this section on, we replace Assumption 1 by the following.

Assumption 4 We consider admissible combinations $(\mathbb{C}>\mathbb{S})$ where the simple model is given by $\mathbb{S}=0,1$.

In this situation, the interface variables belong to $1 \mathrm{D}$ or $0 \mathrm{D}$ models and, therefore, they belong to finite dimensional spaces. Indeed, $\boldsymbol{\mu}_{\mathbb{S}} \in \boldsymbol{\Lambda}_{\mathbb{S}}=\mathbb{R}^{M}$ and $\boldsymbol{\lambda}_{\mathbb{S}} \in \boldsymbol{\Lambda}_{\mathbb{S}}^{\prime}=\mathbb{R}^{M}$. 
An example is provided by the 3D-1D coupled problem described in Sect. 2.3.

Under Assumption 4, we can characterize the operators in (36) through the corresponding matrices, yielding

$$
\left(\begin{array}{cc}
S_{\Gamma_{\mathbb{S}}, \mathbb{S}} & \mathbf{1} \\
S_{\Gamma_{\mathbb{S}}, \mathbb{C}} & -1
\end{array}\right)\left(\begin{array}{l}
\mu_{\mathbb{S}} \\
\lambda_{\mathbb{S}}
\end{array}\right)=\left(\begin{array}{l}
g_{\Gamma_{\mathbb{S}}, \mathbb{S}} \\
g_{\Gamma_{\mathbb{S}}, \mathbb{C}}
\end{array}\right),
$$

where $S_{\Gamma_{\mathbb{S}}, \mathbb{S}}, S_{\Gamma_{\mathbb{S}}, \mathbb{C}}, \mathbf{1} \in \mathbb{R}^{M \times M}$ and $\boldsymbol{g}_{\Gamma_{\mathbb{S}}, \mathbb{S}}, \boldsymbol{g}_{\Gamma_{\mathbb{S}}, \mathbb{C}} \in \mathbb{R}^{M}$.

Similarly, we can write the system of equations when we employ Dirichlet (for the $\mathbb{S D}$ model) and Neumann (for the $\mathbb{C D}$ model) boundary conditions, or Neumann conditions for both models, leading, respectively, to

$$
\left(\begin{array}{cc}
S_{\Gamma_{\mathbb{S}}, \mathbb{S}} & \mathbf{1} \\
\mathbf{1} & -\boldsymbol{T}_{\Gamma_{\mathbb{S}}, \mathbb{C}}
\end{array}\right)\left(\begin{array}{l}
\boldsymbol{\mu}_{\mathbb{S}} \\
\lambda_{\mathbb{S}}
\end{array}\right)=\left(\begin{array}{l}
\boldsymbol{g}_{\Gamma_{\mathbb{S}}, \mathbb{S}} \\
\boldsymbol{R}_{\mathbb{S}} \boldsymbol{u}_{\mathbb{C}}^{J}
\end{array}\right),
$$

and

$$
\left(\begin{array}{cc}
\mathbf{1} & -\boldsymbol{T}_{\Gamma_{\mathbb{S}}, \mathbb{S}} \\
\mathbf{1} & -\boldsymbol{T}_{\Gamma_{\mathbb{S}}, \mathbb{C}}
\end{array}\right)\left(\begin{array}{c}
\boldsymbol{\mu}_{\mathbb{S}} \\
\lambda_{\mathbb{S}}
\end{array}\right)=\left(\begin{array}{c}
\boldsymbol{u}_{\mathbb{S}}^{J} \\
\boldsymbol{R}_{\mathbb{S}} \boldsymbol{u}_{\mathbb{C}}^{J}
\end{array}\right)
$$

These expressions are analogous to (32) and (34), respectively, for which it is also $\boldsymbol{T}_{\Gamma_{\mathbb{S}}, \mathbb{S}}, \boldsymbol{T}_{\Gamma_{\mathbb{S}}, \mathbb{C}} \in \mathbb{R}^{M \times M}$.

Remark 7 Once the physical system is defined we have proper matrices corresponding to each of the augmented problems (Eqs. 38, 39 and 40). It is interesting to investigate the relation between the condition number of those matrices and the number of unknowns in the problem, that is the number of coupling interfaces between $\mathbb{C D}$ models and SD-models. As we will see in Sects. 5 and 6, the condition numbers are increasing functions of the number of coupling interfaces $M$. This feature becomes relevant when attempting to solve the heterogeneous problem in a segregated manner by solving iteratively dimensionally-homogeneous sub-problems.

\section{Discrete dimensionally-heterogeneous problem}

Suppose now that we approximate each component in the system by a Galerkin finite element discretization. We denote by $h_{\mathbb{C}, i}, i=1, \ldots, N_{\mathbb{C}}$, and $h_{\mathbb{S}, j}, j=1, \ldots, N_{\mathbb{S}}$ the characteristic sizes of the elements used in the discretizations of the $\mathbb{C D}$ and $\mathbb{S D}$ subdomains, and $h=\left(h_{\mathbb{C}}, h_{\mathbb{S}}\right)$, where $h_{\mathbb{C}}=\left\{h_{\mathbb{C}, i}\right\}_{i=1}^{N_{\mathbb{C}}}$ and $h_{\mathbb{S}}=\left\{h_{\mathbb{S}, j}\right\}_{j=1}^{N_{\mathbb{S}}}$. Then, the approximate augmented interface problem reads: given $\boldsymbol{u}_{\mathbb{C}, h_{\mathbb{C}}}^{I}$ and $\boldsymbol{u}_{\mathbb{S}, h_{\mathbb{S}}}^{I}$, find $\left(\boldsymbol{\mu}_{\mathbb{S}, h}, \boldsymbol{\lambda}_{\mathbb{S}, h}\right) \in \boldsymbol{\Lambda}_{\mathbb{S}, h} \times \boldsymbol{\Lambda}_{\mathbb{S}, h}^{\prime}$ such that

$$
\begin{array}{ll}
\boldsymbol{s}_{\Gamma_{\mathbb{S}}, \mathbb{S}}\left(\boldsymbol{\mu}_{\mathbb{S}, h}, \hat{\boldsymbol{\mu}}_{\mathbb{S}, h}^{1}\right)+\left\langle\lambda_{\mathbb{S}, h}, \hat{\boldsymbol{\mu}}_{\mathbb{S}, h}^{1}\right\rangle_{\mathbb{S}}=\boldsymbol{g}_{\Gamma_{\mathbb{S}}, \mathbb{S}}\left(\hat{\boldsymbol{\mu}}_{\mathbb{S}, h}^{1}\right) & \forall \hat{\boldsymbol{\mu}}_{\mathbb{S}, h}^{1} \in \boldsymbol{\Lambda}_{\mathbb{S}, h}, \\
\boldsymbol{s}_{\Gamma_{\mathbb{S}}, \mathbb{C}}\left(\boldsymbol{\mu}_{\mathbb{S}, h}, \hat{\boldsymbol{\mu}}_{\mathbb{S}, h}^{2}\right)-\left\langle\lambda_{\mathbb{S}, h}, \hat{\boldsymbol{\mu}}_{\mathbb{S}, h}^{2}\right\rangle_{\mathbb{S}}=\boldsymbol{g}_{\Gamma_{\mathbb{S}}, \mathbb{C}}\left(\hat{\boldsymbol{\mu}}_{\mathbb{S}, h}^{2}\right) & \forall \hat{\boldsymbol{\mu}}_{\mathbb{S}, h}^{2} \in \boldsymbol{\Lambda}_{\mathbb{S}, h}
\end{array}
$$


Analogously, the discrete Steklov-Poincaré formulation reads as follows

$$
\boldsymbol{s}_{\Gamma_{\mathbb{S}}}\left(\boldsymbol{\mu}_{\mathbb{S}, h}, \hat{\boldsymbol{\mu}}_{\mathbb{S}, h}\right)=\boldsymbol{g}_{\Gamma_{\mathbb{S}}}\left(\hat{\boldsymbol{\mu}}_{\mathbb{S}, h}\right) \quad \forall \hat{\boldsymbol{\mu}}_{\mathbb{S}, h} \in \boldsymbol{\Lambda}_{\mathbb{S}, h}
$$

In the case $\mathbb{S}=2$, we should construct suitable conforming finite element spaces to approximate $\boldsymbol{\Lambda}_{\mathbb{S}}$ and $\boldsymbol{\Lambda}_{\mathbb{S}}^{\prime}$ to guarantee the well-posedness of (41). More precisely, we would have to choose a suitable pair $\boldsymbol{\Lambda}_{\mathbb{S}, h} \times \boldsymbol{\Lambda}_{\mathbb{S}, h}^{\prime}$ to be able to prove the discrete counterpart of Proposition 3, that is that there exists a unique pair $\left(\boldsymbol{\mu}_{\mathbb{S}, h}, \boldsymbol{\lambda}_{\mathbb{S}, h}\right) \in \boldsymbol{\Lambda}_{\mathbb{S}, h} \times \boldsymbol{\Lambda}_{\mathbb{S}, h}^{\prime}$ solution of (41) and that there exists $C>0$ such that the solution satisfies

$$
\left\|\boldsymbol{\mu}_{\mathbb{S}, h}\right\|_{\boldsymbol{\Lambda}_{\mathbb{S}, h}}+\left\|\lambda_{\mathbb{S}, h}\right\|_{\boldsymbol{\Lambda}_{\mathbb{S}, h}^{\prime}} \leq C\left(\left\|\boldsymbol{g}_{\Gamma_{\mathbb{S}}, \mathbb{S}, h}\right\|_{\boldsymbol{\Lambda}_{\mathbb{S}, h}^{\prime}}+\left\|\boldsymbol{g}_{\Gamma_{\mathbb{S}}, \mathbb{C}, h}\right\|_{\boldsymbol{\Lambda}_{\mathbb{S}, h}^{\prime}}\right)
$$

The construction of such spaces is not straightforward and it would lead to a too wide discussion that goes beyond the aim of this work. For this reason, we stick to Assumption 4 so that we work only with $\boldsymbol{\Lambda}_{\mathbb{S}}=\boldsymbol{\Lambda}_{\mathbb{S}}^{\prime}=\boldsymbol{\Lambda}_{\mathbb{S}, h}=\boldsymbol{\Lambda}_{\mathbb{S}, h}^{\prime}=\mathbb{R}^{M}$.

Thus, the discrete version of Eq. (41) in block operator form reads: find ( $\boldsymbol{\mu}_{\mathbb{S}, h}$, $\left.\lambda_{\mathbb{S}, h}\right) \in \mathbb{R}^{M} \times \mathbb{R}^{M}$ such that

$$
\left(\begin{array}{cc}
S_{\Gamma_{\mathbb{S}}, \mathbb{S}, h} & \mathbf{1} \\
S_{\Gamma_{\mathbb{S}}, \mathbb{C}, h} & -\mathbf{1}
\end{array}\right)\left(\begin{array}{c}
\boldsymbol{\mu}_{\mathbb{S}, h} \\
\lambda_{\mathbb{S}, h}
\end{array}\right)=\left(\begin{array}{l}
\boldsymbol{g}_{\Gamma_{\mathbb{S}}, \mathbb{S}, h} \\
\boldsymbol{g}_{\Gamma_{\mathbb{S}}, \mathbb{C}, h}
\end{array}\right),
$$

whereas the analogous to (42) is: find $\boldsymbol{\mu}_{\mathbb{S}, h} \in \mathbb{R}^{M}$ such that

$$
\boldsymbol{S}_{\Gamma_{\mathbb{S}}, h} \boldsymbol{\mu}_{\mathbb{S}, h}=\boldsymbol{g}_{\Gamma_{\mathbb{S}}, h} .
$$

Concerning the well-posedness of these problems we can state the following result which is a particular case of Proposition 3.

Proposition 7 There exists a unique pair $\left(\mu_{\mathbb{S}, h}, \lambda_{\mathbb{S}, h}\right) \in \mathbb{R}^{M} \times \mathbb{R}^{M}$ solution of (43). Also, there exists $C>0$ such that the solution satisfies

$$
\left|\boldsymbol{\mu}_{\mathbb{S}, h}\right|+\left|\lambda_{\mathbb{S}, h}\right| \leq C\left(\left|\boldsymbol{g}_{\Gamma_{\mathbb{S}, \mathbb{S}, h}}\right|+\left|\boldsymbol{g}_{\Gamma_{\mathbb{S}}, \mathbb{C}, h}\right|\right)
$$

Proof We have only to prove the analogous of Proposition 1, then the thesis follows from Proposition 3. We show this result for one component, i.e. we fix $j, k$ and $m$. More precisely, we prove in the continuous case that the following problem is well-posed: find $\left(\mathcal{D}_{\mathbb{C}} \mu_{\mathbb{S}}, \lambda_{\mathbb{S}}\right) \in \hat{U}_{\mathbb{C}} \times \mathbb{R}$ such that

$$
\begin{aligned}
a_{\mathbb{C}}\left(\mathcal{D}_{\mathbb{C}} \mu_{\mathbb{S}}, \hat{u}_{\mathbb{C}}^{I}\right)+\left\langle\lambda_{\mathbb{S}}, \mathcal{R}_{\mathbb{S}} \hat{u}_{\mathbb{C}}^{I}\right\rangle_{\mathbb{S}} & =0 \quad \forall \hat{u}_{\mathbb{C}}^{I} \in \hat{U}_{\mathbb{C}}, \\
\left\langle\hat{\lambda}_{\mathbb{S}}, \mathcal{R}_{\mathbb{S}}\left(\mathcal{D}_{\mathbb{C}} \mu_{\mathbb{S}}\right)\right\rangle_{\mathbb{S}} & =\left\langle\hat{\lambda}_{\mathbb{S}}, \mu_{\mathbb{S}}\right\rangle_{\mathbb{S}} \quad \forall \hat{\lambda}_{\mathbb{S}} \in \mathbb{R} .
\end{aligned}
$$

Notice that in this context the duality pairing $\langle\cdot, \cdot\rangle_{\mathbb{S}}$ reduces to the Euclidean scalar product in $\mathbb{R}$.

Let $w_{\mathbb{C}} \in \hat{U}_{\mathbb{C}}$ be the solution of the following problem:

$$
a_{\mathbb{C}}\left(w_{\mathbb{C}}, \hat{u}_{\mathbb{C}}^{I}\right)=-\left\langle 1, \mathcal{R}_{\mathbb{S}} \hat{u}_{\mathbb{C}}^{I}\right\rangle_{\mathbb{S}} \quad \forall \hat{u}_{\mathbb{C}}^{I} \in \hat{U}_{\mathbb{C}}
$$


Then $\mathcal{D}_{\mathbb{C}} \mu_{\mathbb{S}}=\lambda_{\mathbb{S}} w_{\mathbb{C}}$ satisfies the first equation in (44). If we require that it satisfies also the second equation, we obtain:

$$
\lambda_{\mathbb{S}}\left\langle\hat{\lambda}_{\mathbb{S}}, \mathcal{R}_{\mathbb{S}} w_{\mathbb{C}}\right\rangle_{\mathbb{S}}=\left\langle\hat{\lambda}_{\mathbb{S}}, \mu_{\mathbb{S}}\right\rangle_{\mathbb{S}} \quad \forall \hat{\lambda}_{\mathbb{S}} \in \mathbb{R}
$$

Thanks to the coercivity of the bilinear form $a_{\mathbb{C}}(\cdot, \cdot)$, from (45) it follows that $\left\langle\hat{\lambda}_{\mathbb{S}}\right.$, $\left.\mathcal{R}_{\mathbb{S}} w_{\mathbb{C}}\right\rangle_{\mathbb{S}} \neq 0$ whenever $\hat{\lambda}_{\mathbb{S}} \neq 0$, so that $\lambda_{\mathbb{S}}$ exists.

To prove uniqueness, let $\left(u_{\mathbb{C}}^{1}, \lambda_{\mathbb{S}}^{1}\right),\left(u_{\mathbb{C}}^{2}, \lambda_{\mathbb{S}}^{2}\right) \in \hat{U}_{\mathbb{C}} \times \mathbb{R}$ be two solutions of (44). Then, there holds:

$$
\begin{aligned}
a_{\mathbb{C}}\left(u_{\mathbb{C}}^{1}-u_{\mathbb{C}}^{2}, \hat{u}_{\mathbb{C}}^{I}\right)+\left\langle\lambda_{\mathbb{S}}^{1}-\lambda_{\mathbb{S}}^{2}, \mathcal{R}_{\mathbb{S}} \hat{u}_{\mathbb{C}}^{I}\right\rangle_{\mathbb{S}}=0 & \forall \hat{u}_{\mathbb{C}}^{I} \in \hat{U}_{\mathbb{C}}, \\
\left\langle\hat{\lambda}_{\mathbb{S}}, \mathcal{R}_{\mathbb{S}}\left(u_{\mathbb{C}}^{1}-u_{\mathbb{C}}^{2}\right)\right\rangle_{\mathbb{S}}=0 & \forall \hat{\lambda}_{\mathbb{S}} \in \mathbb{R} .
\end{aligned}
$$

Taking $\hat{u}_{\mathbb{C}}^{I}=u_{\mathbb{C}}^{1}-u_{\mathbb{C}}^{2}$, by coercivity of $a_{\mathbb{C}}(\cdot, \cdot)$ we obtain $\left\|u_{\mathbb{C}}^{1}-u_{\mathbb{C}}^{2}\right\|_{U_{\mathbb{C}}}=0$ from which $u_{\mathbb{C}}^{1}=u_{\mathbb{C}}^{2}$ a.e. in $\Omega_{\mathbb{C}}$. The equality $\lambda_{\mathbb{S}}^{1}=\lambda_{\mathbb{S}}^{2}$ follows straightforwardly.

Finally, concerning the conditioning of the problem, we have the following result.

Proposition 8 The condition number of matrices $S_{\Gamma_{\mathbb{S}}, h}$ and $\left(\begin{array}{cc}S_{\Gamma_{\mathbb{S}}, \mathbb{S}, h} & 1 \\ S_{\Gamma_{\mathbb{S}}, C, h} & -1\end{array}\right)$ is independent of $h=\left(h_{\mathbb{C}}, h_{\mathbb{S}}\right)$.

Proof The proof is by contradiction. Consider matrix $S_{\Gamma_{\mathbb{S}}, h}$. Assume that the condition number depends on a negative power of $h$. Note that the space where the approximate solution is looked for does not depend on $h=\left(h_{\mathbb{C}}, h_{\mathbb{S}}\right)$. Indeed, the space where both the exact and the approximate solutions live is exactly the same. Since the operator $\mathcal{S}_{\Gamma_{\mathbb{S}}, h}$ is invertible, the matrix $\boldsymbol{S}_{\Gamma_{\mathbb{S}}, h}$ associated to the discrete problem is also invertible. The independence of the condition number of the system with respect to parameter $h$ stems from the following argument. The matrix $S_{\Gamma_{\mathbb{S}}, h}$ of the continuous operator has a condition number $K\left(S_{\Gamma_{\mathbb{S}}, h}\right)$ that obviously does not depend on $h$. Therefore, for $h \rightarrow 0$, we have

$$
S_{\Gamma_{\mathbb{S}}, h} \rightarrow S_{\Gamma_{\mathbb{S}}} \text { and } \quad K\left(S_{\Gamma_{\mathbb{S}}, h}\right) \rightarrow K\left(S_{\Gamma_{\mathbb{S}}}\right)
$$

The former is a convergence in $\mathbb{R}^{M \times M}$, so the analysis is straightforward and we can conclude by contradiction that $K\left(S_{\Gamma_{\mathbb{S}}, h}\right)$ does not depend on $h$. For the case of block matrix $\left(\begin{array}{cc}S_{\Gamma_{\mathbb{S}}, \mathbb{S}, h} & 1 \\ S_{\Gamma_{\mathbb{S}}, \mathbb{C}, h} & -1\end{array}\right)$ the same arguments hold and the result follows.

Remark 8 In a completely analogous way, it can be seen that the discrete versions of the problems defined by Eqs. (39) and (40) enjoy the same property. That is, the condition number of block matrices $\left(\begin{array}{cc}\boldsymbol{S}_{\Gamma_{\mathbb{S}}, \mathbb{S}, h} & \mathbf{1} \\ \mathbf{1} & -\boldsymbol{T}_{\Gamma_{\mathbb{S}}, \mathbb{C}, h}\end{array}\right)$ and $\left(\begin{array}{ll}\mathbf{1} & -\boldsymbol{T}_{\Gamma_{\mathbb{S}}, \mathbb{S}, h} \\ \mathbf{1} & -\boldsymbol{T}_{\Gamma_{\mathbb{S}}, \mathbb{C}, h}\end{array}\right)$ is independent of $h=\left(h_{\mathbb{C}}, h_{\mathbb{S}}\right)$.

\section{Numerical experiments}

In this section we present two applications of our theory. Particularly, we provide numerical evidence to the conclusions drawn in Remark 7, and Proposition 8. The 


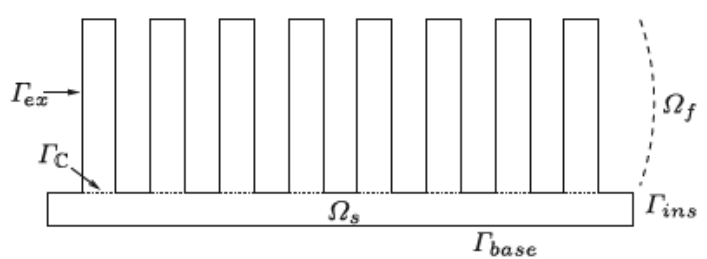

Fig. 5 Schematic representation of a thermal fin

applications considered are the heat transfer problem with pure diffusion phenomena and the linear elasticity problem.

\subsection{Coupling 2D-1D systems: heat transfer}

In this example, we consider a 2D heat sink designed for the thermal management of high-density electronic components, formed by a base or spreader which supports a number of plate fins exposed to flowing air (see [21]). A schematic representation of the computational domain is presented in Figure 5.

The system is described by the following equations:

$$
\begin{array}{ll}
-\operatorname{div}\left(k \nabla u_{2}\right)=0 & \text { in } \Omega_{s} \cup \Omega_{f}, \\
k \frac{\partial u_{2}}{\partial n}=0 & \text { on } \Gamma_{\text {ins }}, \\
u_{2}=u_{2}^{*} & \text { on } \Gamma_{\text {base }}, \\
k \frac{\partial u_{2}}{\partial n}+\operatorname{Bi} u_{2}=0 & \text { on } \Gamma_{e x},
\end{array}
$$

where $k$ is the adimensional thermal conductivity: $k=k_{s} / k_{f}, k_{s}$ and $k_{f}$ being the thermal conductivities of the spreader and of the fin, respectively. Finally, $\mathrm{Bi}$ is the adimensional Biot number: $\mathrm{Bi}=h_{c} d_{\text {per }} / k_{f}$ where $h_{c}$ is the heat transfer coefficient and $d_{p e r}$ the distance between the fins. $u_{2}$ represents the adimensional temperature inside the heat sink.

On the interfaces $\Gamma_{\mathbb{C}}$ we impose the continuity of the mean temperature and that of the heat fluxes.

For large systems of thermal fins, in order to reduce the computational cost, one may replace the fins by $1 \mathrm{D}$ structures. This approximation is significant especially when the Biot number is small, which corresponds to a temperature distribution in the fins which behaves almost as a 1D distribution.

In such a case, according to the notation introduced in this work, we have $\mathbb{S}=$ $1, \mathbb{C}=2, \Omega_{1}$ is the domain of $1 \mathrm{D}$ fins (with coordinate $\xi$ ) while $\Omega_{2}$ is the domain made of the spreader and possible 2D fins (with coordinates $(x, y)$ ). Moreover, $\Gamma_{1}$ and $\Gamma_{2}$ are the 1D and 2D coupling interfaces, respectively.

The linear manifolds become $U_{2}=H^{1}\left(\Omega_{2}\right)+$ b.c. and $U_{1}=H^{1}\left(\Omega_{1}\right)+$ b.c., while we have $\Lambda_{2}=H^{1 / 2}\left(\Gamma_{2}\right), \Lambda_{2}^{\prime}=H^{-1 / 2}\left(\Gamma_{2}\right), \Lambda_{1}=\Lambda_{1}^{\prime}=\mathbb{R}$. The problem is defined 


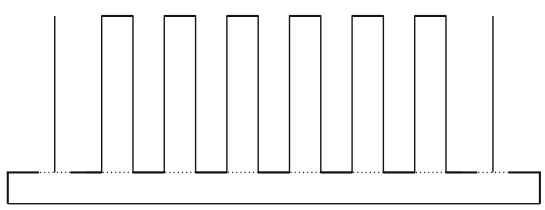

$21 \mathrm{D}$ fins

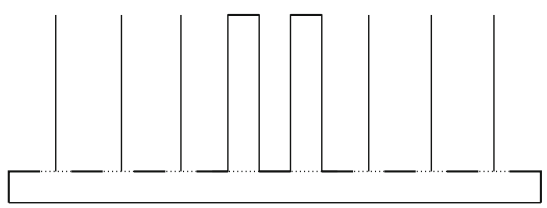

6 1D fins

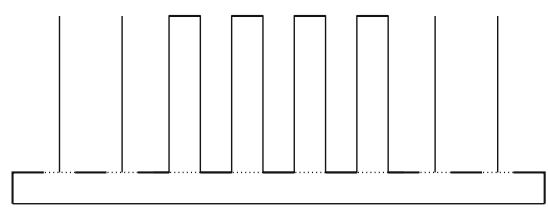

4 1D fins

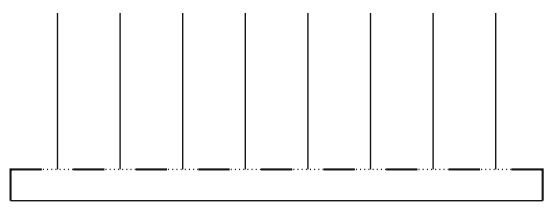

$81 \mathrm{D}$ fins

Fig. 6 Different heterogeneous configurations for the same physical system

by the following continuous and coercive bilinear forms:

$$
\begin{aligned}
& a_{2}\left(u_{2}, \hat{u}_{2}\right)=\int_{\Omega_{2}} k \nabla u_{2} \cdot \nabla \hat{u}_{2} \mathrm{~d} \Omega_{2}+\int_{\Gamma_{e x}} \operatorname{Bi} u_{2} \hat{u}_{2} \mathrm{~d} \Gamma_{e x}, \\
& a_{1}\left(u_{1}, \hat{u}_{1}\right)=\int_{\Omega_{1}} k \delta \frac{\mathrm{d} u_{1}}{\mathrm{~d} \xi} \frac{\mathrm{d} \hat{u}_{1}}{\mathrm{~d} \xi} \mathrm{d} \Omega_{1}+\int_{\Omega_{1}} 2 \operatorname{Bi} u_{1} \hat{u}_{1} \mathrm{~d} \Omega_{1},
\end{aligned}
$$

where $\delta$ is the width of the fins.

The operators $\mathcal{R}_{1}$ and $\mathcal{R}_{1}^{*}$ are defined as

$$
\mathcal{R}_{1}\left(u_{2 \mid \Gamma_{2}}\right)=u_{2,1 \mid \Gamma_{1}}=\frac{1}{\left|\Gamma_{2}\right|} \int_{\Gamma_{2}} u_{2} \mathrm{~d} \Gamma_{2} \quad \text { and } \quad \mathcal{R}_{1}^{*}\left(\lambda_{1}\right)=\lambda_{1 \mid \Gamma_{2}}
$$

and we have

$$
\begin{aligned}
\left\langle\lambda_{1}, u_{1}\right\rangle_{1} & =\left|\Gamma_{2}\right| \lambda_{1} u_{1 \mid \Gamma_{1}}, \\
\left\langle\lambda_{1}, \mathcal{R}_{1}\left(u_{2 \mid \Gamma_{2}}\right)\right\rangle_{1} & =\left|\Gamma_{2}\right| \lambda_{1} u_{2,1 \mid \Gamma_{1}}=\int_{\Gamma_{2}} \lambda_{1 \mid \Gamma_{2}} u_{2} \mathrm{~d} \Gamma_{2}=\left\langle\mathcal{R}_{1}^{*}\left(\lambda_{1}\right), u_{2}\right\rangle_{2} .
\end{aligned}
$$

Following the same steps of Remark 2, it can be easily seen that these operators satisfy the hypotheses of Proposition 1.

We solve the coupled problem by considering the four configurations shown in Fig. 6. In the first case we have $M=2$ interfaces so that the augmented system has dimension $4 \times 4$, in the second case $M=4$ corresponding to a $8 \times 8$ system, while in the last two cases $M=6$ and 8 , respectively, corresponding to augmented systems of dimensions 12 and 16 .

We use the Dirichlet-and-Neumann and the Neumann-and-Neumann approaches (39) and (40), respectively. 
Table 1 Number of degrees of freedom for the different configurations

\begin{tabular}{|c|c|c|c|c|c|c|c|c|}
\hline & \multicolumn{2}{|l|}{2 1D fins } & \multicolumn{2}{|l|}{4 1D fins } & \multicolumn{2}{|l|}{6 1D fins } & \multicolumn{2}{|l|}{8 1D fins } \\
\hline & dofs $\Omega_{1}$ & dofs $\Omega_{2}$ & dofs $\Omega_{1}$ & dofs $\Omega_{2}$ & dofs $\Omega_{1}$ & dofs $\Omega_{2}$ & dofs $\Omega_{1}$ & dofs $\Omega_{2}$ \\
\hline grid 1 & 22 & 165 & 44 & 137 & 66 & 109 & 88 & 81 \\
\hline grid 2 & 42 & 537 & 84 & 449 & 126 & 361 & 168 & 273 \\
\hline grid 3 & 82 & 1,905 & 164 & 1,601 & 246 & 1,297 & 328 & 993 \\
\hline grid 4 & 162 & 7,137 & 324 & 6,017 & 486 & 4,897 & 648 & 3,777 \\
\hline grid 5 & 322 & 27,585 & 644 & 23,297 & 966 & 19,009 & 1,288 & 14,721 \\
\hline
\end{tabular}

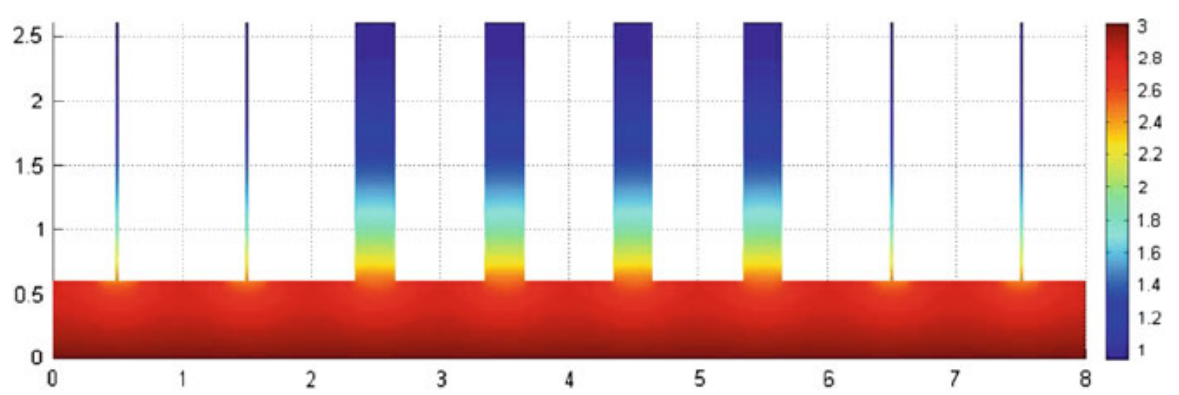

Fig. 7 Solution computed for the second configuration with 4 1D fins

For our simulations we consider $k=1, u_{2}^{*}=3, \mathrm{Bi}=0.1$ and $\delta=0.3$. We carry out a finite element discretization considering $\mathbb{P}_{1}$ Lagrangian elements and several computational grids depending on $h_{1}$ and $h_{2}$ as shown in Table 1 .

In Fig. 7 we show the solution computed for the second configuration, while in Fig. 8 we compare the solution on one of the fins using two different configurations (those with 4 and 6 1D fins) corresponding to treating that fin as a 1D or as a 2D model. Finally, in Table 2 we report the condition numbers of the augmented systems and the number of iterations required to converge. Despite their small dimensions, the linear systems have been solved using BiCGStab iterations (with tolerance $10^{-6}$ on the relative residual) to avoid computing explicitly the Dirichlet-to-Neumann or Neumann-to-Dirichlet operators for the 2D problem.

As pointed out in Proposition 8, we can observe that the condition numbers are independent of both $h_{1}$ and $h_{2}$ and, although mildly, the condition number grows with $M$.

\subsection{Coupling 3D-1D systems: linear elasticity}

In this section we consider the problem of a linear elastic body governed by the Navier equations. Particularly, we perform the analysis of a structural component in the frequency domain, for which we make use of the frequency domain equations, which are called reduced field equations of elastodynamics [11]. In this case we assume that the boundary conditions are harmonic in time with angular velocity $\omega$. Let us assume that 


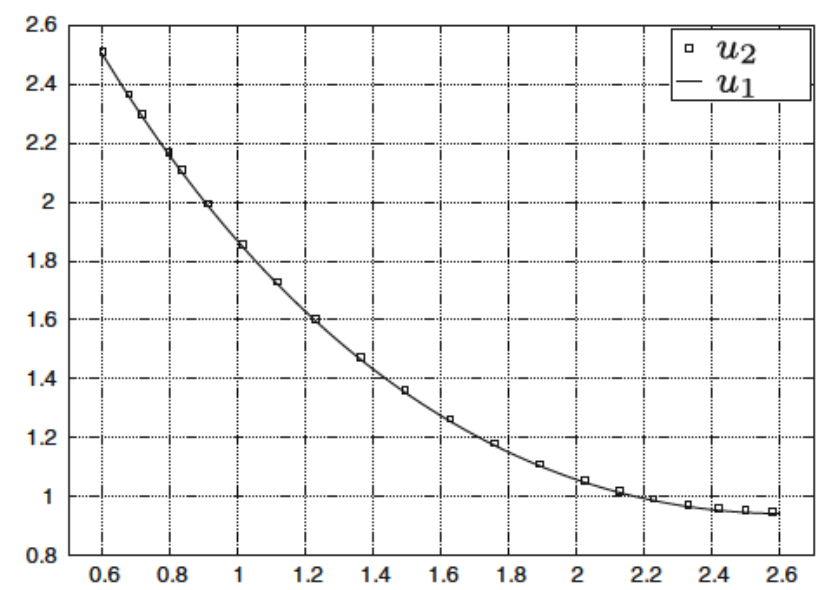

Fig. 8 Temperatures computed with the 1D model (solid line) and mean values obtained from the 2D model (squares) for a sample fin, using the configurations with 4 and $61 \mathrm{D}$ fins, respectively

Table 2 Condition numbers and number of iterations (between brackets) for the Dirichlet-and-Neumann method (left) and for the Neumann-and-Neumann method (right)

\begin{tabular}{|c|c|c|c|c|c|c|c|c|}
\hline & \multicolumn{4}{|c|}{ Dirichlet-and-Neumann method } & \multicolumn{4}{|c|}{ Neumann-and-Neumann method } \\
\hline & 2 & & & & & & & ins \\
\hline grid 1 & & & $3.1799(6)$ & 3.1994 (5) & $2.0014(3)$ & $2.0529(4)$ & $2.0708(4)$ & $2.0095(4)$ \\
\hline $\mathrm{d} 2$ & 3.068 & 1 & 3 & 3 . & 1. & 4) & 1.9 & 2.0 \\
\hline id 3 & 3.0549 & 3.0970 (4) & & & & & & $1.9838(4)$ \\
\hline s & $3.0506(4)$ & $3.0923(4)$ & 3.1072 & $3.1211(4)$ & & & $1.9615(4)$ & $1.9748(3)$ \\
\hline id 5 & $3.0493(3)$ & 3.0909 (4) & $3.1058(5)$ & 3.1195 (4) & $1.9014(2)$ & 1.94 & $9(4)$ & 1.9721( \\
\hline
\end{tabular}

the mechanism is endowed with a continuously-distributed kinematic linear control system.

In view of the geometrical characteristics of the mechanism under study we construct a representation through coupled dimensionally-heterogeneous 3D-1D models as shown in Fig. 9. The mechanism consists of one centered 3D model, four cornered 3D models, four diagonal 1D bars connecting the centered 3D model to the cornered ones and four in-plane 1D bars connecting the cornered 3D models among them. In this example we have that $M=16$ is the number of coupling interfaces, so the dimension of the interface problem is $2 M=32$. The structure is component-wise homogeneous, since the $1 \mathrm{D}$ bars have a different material parameter than the 3D components.

According to the notation introduced so far, the computational model for this problem is characterized by being $\mathbb{S}=1$ and $\mathbb{C}=3$ (recall that $\alpha=1$ ), for which $\Omega_{1}$ is the domain of the bar components (with coordinate $\xi$ ) and $\Omega_{3}$ is the 3D domain of the solid components (with coordinates $(x, y, z)$ ). Also, $\Gamma_{1}$ is the 1D coupling interface (point) and $\Gamma_{3}$ is the 3D coupling interface (planar surface) with outward unit normal $\mathbf{n}$ (which coincides with the axial direction of the bar). The linear manifolds are 

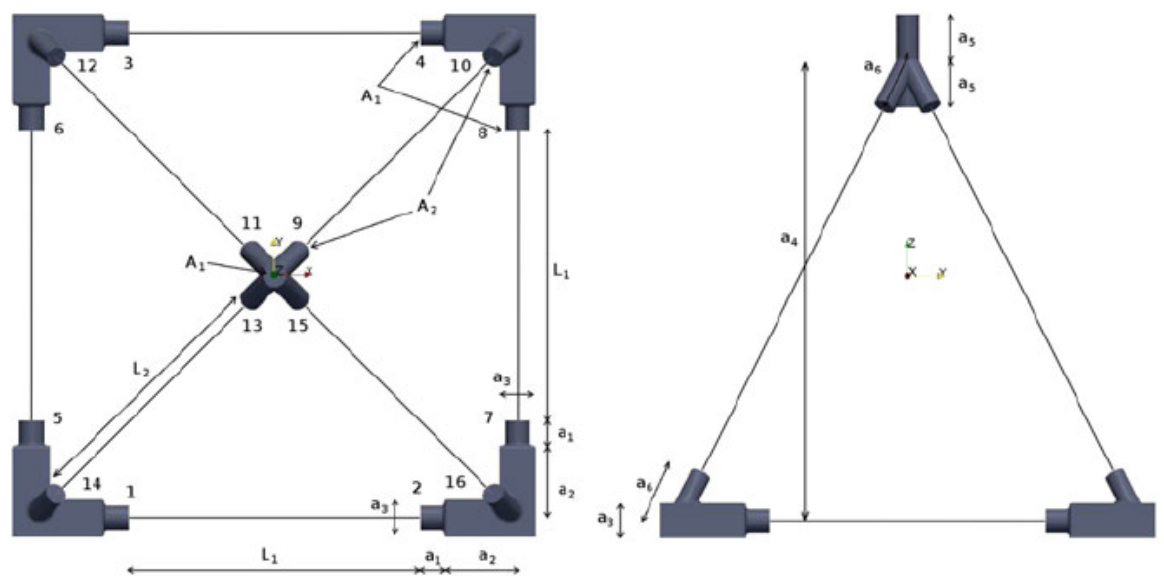

Fig. 9 Structural mechanism modeled by means of 3D-1D coupled models

$U_{3}=\mathbf{H}^{1}\left(\Omega_{3}\right)+$ b.c. and $U_{1}=H^{1}\left(\Omega_{1}\right)+$ b.c., while it is $\Lambda_{3}=\mathbf{H}^{1 / 2}\left(\Gamma_{3}\right), \Lambda_{3}^{\prime}=$ $\mathbf{H}^{-1 / 2}\left(\Gamma_{3}\right)$ and $\Lambda_{1}=\Lambda_{1}^{\prime}=\mathbb{R}$. Here $u_{1}$ denotes the axial displacement in the 1D bar and $\mathbf{u}_{3}$ is the displacement field (vector field) in the 3D domain.

When incorporating the distributed kinematic control system, the bilinear and linear forms for the reduced field equations are as follows

$$
\begin{aligned}
a_{1}\left(u_{1}, \hat{u}_{1}\right) & =\int_{\Omega_{1}}\left(K-\rho \omega^{2}\right) A u_{1} \hat{u}_{1} \mathrm{~d} \Omega_{1}+\int_{\Omega_{1}} A \tilde{E} \frac{d u_{1}}{d \xi} \frac{d \hat{u}_{1}}{d \xi} \mathrm{d} \Omega_{1}, \\
a_{3}\left(\mathbf{u}_{3}, \hat{\mathbf{u}}_{3}\right) & =\int_{\Omega_{3}}\left(K-\rho \omega^{2}\right) \mathbf{u}_{3} \cdot \hat{\mathbf{u}}_{3} \mathrm{~d} \Omega_{3}+\int_{\Omega_{3}} \mathbb{E}\left(\nabla \mathbf{u}_{3}\right)^{s} \cdot\left(\nabla \hat{\mathbf{u}}_{3}\right)^{s} \mathrm{~d} \Omega_{3}, \\
f_{1}\left(\hat{u}_{1}\right) & =\int_{\Omega_{1}} A g \hat{u}_{1} \mathrm{~d} \Omega_{1}, \\
f_{3}\left(\hat{\mathbf{u}}_{3}\right) & =\int_{\Omega_{3}} \mathbf{g} \cdot \hat{\mathbf{u}}_{3} \mathrm{~d} \Omega_{3} .
\end{aligned}
$$

The operators $\mathcal{R}_{1}$ and $\mathcal{R}_{1}^{*}$ are defined by

$$
\begin{aligned}
\mathcal{R}_{1}\left(\mathbf{u}_{3 \mid \Gamma_{3}}\right) & =u_{3,1} \mid \Gamma_{1}=\frac{1}{\left|\Gamma_{3}\right|} \int_{\Gamma_{3}} \mathbf{u}_{3} \cdot \mathbf{n} \mathrm{d} \Gamma_{3}, \\
\mathcal{R}_{1}^{*}\left(\lambda_{1}\right) & =\lambda_{1 \mid \Gamma_{3}} \mathbf{n},
\end{aligned}
$$

and the duality pairings are given by

$$
\left\langle\lambda_{1}, u_{1}\right\rangle_{1}=A_{\mid \Gamma_{1}} \lambda_{1} u_{1 \mid \Gamma_{1}},
$$




$$
\left\langle\lambda_{1}, \mathcal{R}_{1}\left(\mathbf{u}_{3 \mid \Gamma_{3}}\right)\right\rangle_{1}=A_{\mid \Gamma_{1}} \lambda_{1} u_{3,1 \mid \Gamma_{1}}=\int_{\Gamma_{3}} \lambda_{1 \mid \Gamma_{3}} \mathbf{n} \cdot \mathbf{u}_{3} \mathrm{~d} \Gamma_{3}=\left\langle\mathcal{R}_{1}^{*}\left(\lambda_{1}\right), \mathbf{u}_{3}\right\rangle_{3}
$$

In the expressions above $A$ is the cross sectional area of the bar, noting that $A_{\mid \Gamma_{1}}=$ $\left|\Gamma_{3}\right|, \mathbb{E}$ is the fourth order elasticity tensor in the solid domain, $\tilde{E}$ is the effective elasticity modulus in the axial direction of the $\operatorname{bar}(\tilde{E}=\mathbb{E} \cdot(\mathbf{n} \otimes \mathbf{n} \otimes \mathbf{n} \otimes \mathbf{n})), \mathbf{g}$ is a volume source in the solid domain, while $g$ is a volume source in the axial direction of the bar $(g=\mathbf{g} \cdot \mathbf{n})$. As well, $K$ is responsible for the linear control system acting in a distributed manner over the mechanism. Here the parameters are set always such that $K-\rho \omega^{2}>0$.

Evidently, the forms $a_{1}$ and $a_{3}$ are bilinear, continuous and also coercive, while the forms $f_{1}$ and $f_{3}$ are linear functionals. In turn, the operator $\mathcal{R}_{1}$ is linear and continuous, while its transpose $\mathcal{R}_{1}^{*}$ satisfies the requirements stated in Proposition 1 [see inequalities (12)]. As a matter of fact, for the right inequality we have

$$
\begin{aligned}
\left\|\mathcal{R}_{1}^{*} \lambda_{1}\right\|_{\mathbf{H}^{-1 / 2}\left(\Gamma_{3}\right)} & =\sup _{\mathbf{u}_{3} \in \mathbf{H}^{1 / 2}\left(\Gamma_{3}\right)} \frac{\mathbf{H}^{-1 / 2}\left(\Gamma_{3}\right)}{\left.\| \mathcal{R}_{1}^{*} \lambda_{1}, \mathbf{u}_{3}\right\rangle_{\mathbf{H}^{1 / 2}\left(\Gamma_{3}\right)}} \\
& =\sup _{\mathbf{u}_{3} \in \mathbf{H}_{3} \|_{\mathbf{H}^{1 / 2}\left(\Gamma_{3}\right)}\left(\Gamma_{3}\right)} \frac{\mathbb{R}\left\langle\lambda_{1}, \mathcal{R}_{1} \mathbf{u}_{3}\right\rangle_{\mathbb{R}}}{\left\|\mathbf{u}_{3}\right\|_{\mathbf{H}^{1 / 2}\left(\Gamma_{3}\right)}}=\left|\lambda_{1}\right| \sup _{\mathbf{u}_{3} \in \mathbf{H}^{1 / 2}\left(\Gamma_{3}\right)} \frac{\left|\mathcal{R}_{1} \mathbf{u}_{3}\right|}{\left\|\mathbf{u}_{3}\right\|_{\mathbf{H}^{1 / 2}\left(\Gamma_{3}\right)}} \\
& =\frac{\left|\lambda_{1}\right|}{\left|\Gamma_{3}\right|} \sup _{\mathbf{u}_{3} \in \mathbf{H}^{1 / 2}\left(\Gamma_{3}\right)} \frac{\int_{\Gamma_{3}} \mathbf{u}_{3} \cdot \mathbf{n} \mathrm{d} \Gamma_{3}}{\left\|\mathbf{u}_{3}\right\|_{\mathbf{H}^{1 / 2}\left(\Gamma_{3}\right)}} \\
& \leq \frac{\left|\lambda_{1}\right|}{\left|\Gamma_{3}\right|} \sup _{\mathbf{u}_{3} \in \mathbf{H}^{1 / 2}\left(\Gamma_{3}\right)} \frac{\left\|\mathbf{u}_{3}\right\|_{\mathbf{L}^{2}\left(\Gamma_{3}\right)}\left|\Gamma_{3}\right|}{\left\|\mathbf{u}_{3}\right\|_{\mathbf{H}^{1 / 2}\left(\Gamma_{3}\right)}} \leq C\left|\lambda_{1}\right| \quad \forall \lambda_{1} \in \mathbb{R} .
\end{aligned}
$$

For the left inequality let us take $\hat{\mathbf{u}}_{3}$ such that $\left|\hat{\mathbf{u}}_{3}\right|=\hat{\mathbf{u}}_{3} \cdot \mathbf{n}=1$, that is, it is a constant function equal to one in the direction of the normal vector. Then $\mathcal{R}_{1} \hat{\mathbf{u}}_{3}=1$ and $\left\|\hat{\mathbf{u}}_{3}\right\|_{\mathbf{H}^{1 / 2}\left(\Gamma_{3}\right)}=1$, hence

$$
\begin{aligned}
\left|\lambda_{1}\right| & =\frac{\left|\mathbb{R}\left\langle\lambda_{1}, \mathcal{R}_{1} \hat{\mathbf{u}}_{3}\right\rangle_{\mathbb{R}}\right|}{\left\|\hat{\mathbf{u}}_{3}\right\|_{\mathbf{H}^{1 / 2}\left(\Gamma_{3}\right)}} \leq \sup _{\mathbf{u}_{3} \in \mathbf{H}^{1 / 2}\left(\Gamma_{3}\right)} \frac{\mathbb{R}\left\langle\lambda_{1}, \mathcal{R}_{1} \mathbf{u}_{3}\right\rangle_{\mathbb{R}}}{\left\|\mathbf{u}_{3}\right\|_{\mathbf{H}^{1 / 2}\left(\Gamma_{3}\right)}} \\
& =\sup _{\mathbf{u}_{3} \in \mathbf{H}^{1 / 2}\left(\Gamma_{3}\right)} \frac{\mathbf{H}^{-1 / 2}\left(\Gamma_{3}\right)}{\left\langle\mathcal{R}_{1}^{*} \lambda_{1}, \mathbf{u}_{3}\right\rangle_{\mathbf{H}^{1 / 2}\left(\Gamma_{3}\right)}}=\left\|\mathcal{R}_{1}^{*} \lambda_{1}\right\|_{\mathbf{H}^{-1 / 2}\left(\Gamma_{3}\right)} \quad \forall \lambda_{1} \in \mathbb{R} .
\end{aligned}
$$

Particularly, for this example we take $g=0, \mathbf{g}=\mathbf{0}, \mathbb{E}$ is characterized by the Young modulus $E_{3 D}=20.0$ and the Poisson ratio $v=0.3$, while $\tilde{E}=\frac{E_{1 D}(1-v)}{(1+v)(1-2 v)}$, being $E_{1 D}=37$ the Young modulus of the $1 \mathrm{D}$ components. The density is $\rho=7.86 \cdot 10^{-6}$ and the control system is characterized by $K=1.0$. 

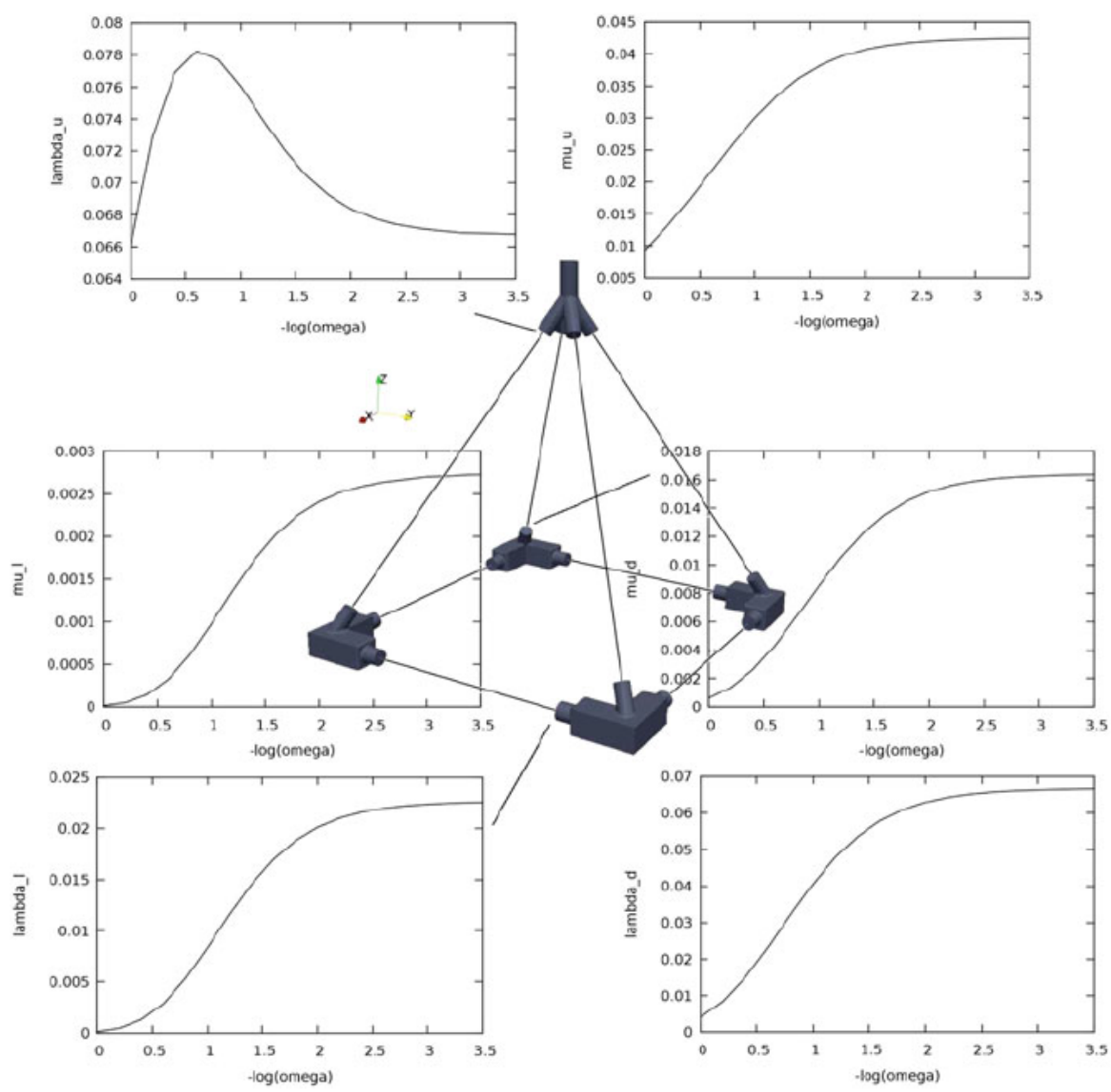

Fig. 10 Results at coupling points $l, d$ and $u$ of the mechanism

The boundary conditions are such that the displacement is prescribed on the upper part of the centered 3D model in Fig. 9, and in the frequency domain its value is $\overline{\mathbf{u}}_{3}=-\delta u \mathbf{e}_{z}, \delta u=0.1$, being $\mathbf{e}_{z}$ the unit vector in the $z$-direction. In addition, the cornered 3D models in the lower part of the mechanism are fixed in the vertical direction and are free in the two in-plane directions, that is in the $(x, y)$-plane. The spatial discretization with the characteristic lengths given by $h_{3}$ and $h_{1}$ are such that the meshes have: 10270 nodes for the centered 3D model, 23305 nodes for the cornered 3D models, 81 nodes for the diagonal 1D bars and 61 nodes for the in-plane 1D bars. The dimensions that define the mechanism are $A_{1}=0.7854, A_{2}=0.6504, L_{1}=12.0, L_{2}=$ $19.5959, a_{1}=1.0, a_{2}=4.0, a_{3}=1.5, a_{4}=20.0, a_{5}=2.0$ and $a_{6}=2.4495$.

In spite of the symmetry of the geometry and of the loading we keep the original structure involving the five 3D solid models and the eight 1D bar models. Particularly, this problem was solved using a Neumann-and-Neumann approach, that is Neumann boundary conditions for all the components according to (40). The linear problem was solved using the Newton method which takes $2 M=32$ iterations to evaluate the 


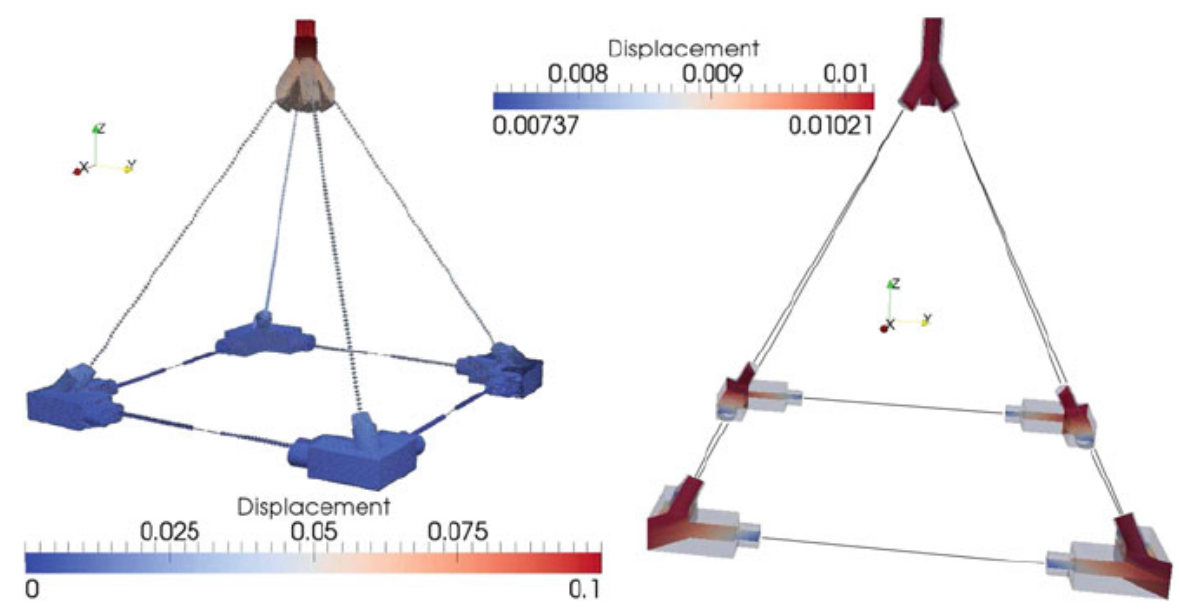

Fig. 11 Displacement field in the mechanism for $-\log (\omega)=3$

Jacobian, where $M$ is the number of coupling interfaces (recall that $M=16$ in this problem).

The frequency analysis performed in the present application entails studying the way in which the coupling quantities $\left(\mu_{1}, \lambda_{1}\right)_{m}, m=1, \ldots, M$ depend upon the frequency $\omega$ of the excitation, that is the frequency of the prescribed displacement over the upper part of the mechanism (over the centered 3D model). Due to the symmetries of the mechanism we have three average displacements and three coupling forces, denoted by $\left(\mu_{l}, \lambda_{l}\right),\left(\mu_{d}, \lambda_{d}\right)$ and $\left(\mu_{u}, \lambda_{u}\right)$. The indexes $l, d$ and $u$ denote the solution at the coupling points which are equivalent, that is $\left(\mu_{l}, \lambda_{l}\right)=\left(\mu_{i}, \lambda_{i}\right), i=$ $1, \ldots, 8,\left(\mu_{d}, \lambda_{d}\right)=\left(\mu_{i}, \lambda_{i}\right), i=10,12,14,16$ and $\left(\mu_{u}, \lambda_{u}\right)=\left(\mu_{i}, \lambda_{i}\right), i=$ $9,11,13,15$ (see Fig. 9 for the numeration of the coupling points).

Figure 10 presents the way in which the coupling quantities at points $l, d$ and $u$ depend on the frequency $\omega$. In both, mean displacement and coupling force, the saturation point is easily noticed when the frequency $\omega$ approaches from the limit value $\sqrt{\frac{K}{\rho}}$. These results are not further discussed because this goes beyond the scope of the present work.

In view of the load acting over the mechanism, the four diagonal bars are in a compression state, and so the sign of the coupling force is such that it is a compressive force, while the in-plane bars are all in a traction state, and therefore the coupling force is indeed a traction force. As a result, the mean displacements are such that the mechanism undergoes a center-to-outer deformation. Figure 11 displays the displacement vector field in the mechanism as well as the solution (magnitude of the displacement field) in some slices cutting the 3D components. In turn, in Fig. 12 the original and deformed configurations are shown, for which an amplification factor has been used over the displacement field. In such figure we can observe what was said above, that is, the traction and compressive states of the bars as a result of the 3D-1D heterogeneous interaction of the entire component. 


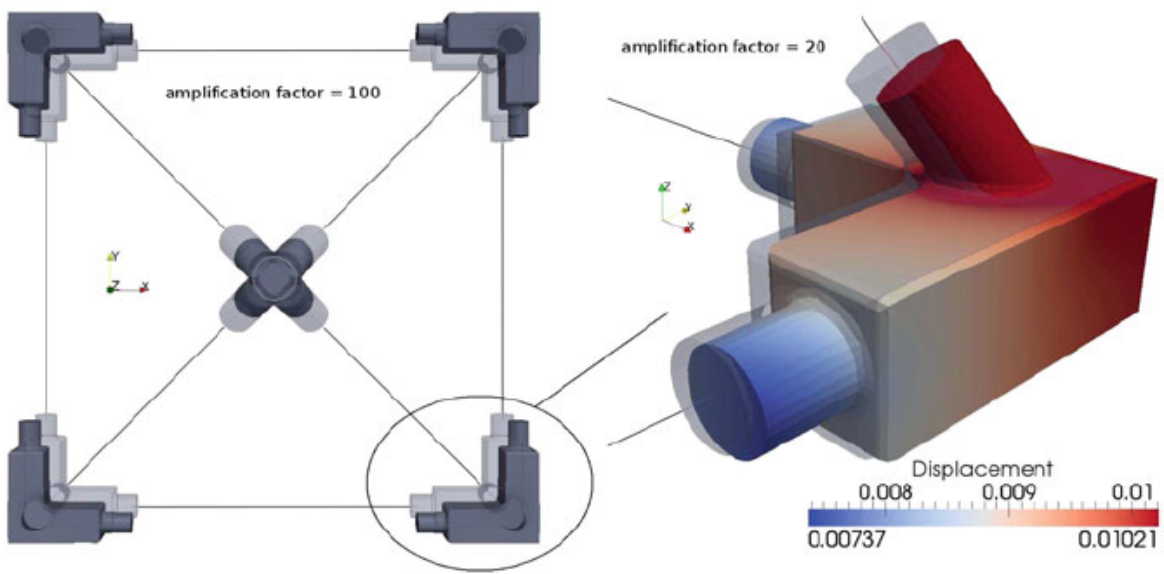

Fig. 12 Original and deformed configuration for $-\log (\omega)=3$ (displacements are amplified)

\section{Conclusions}

In this work, the mathematical framework for coupling dimensionally-heterogeneous models was set up. This was carried out starting from an extended variational formulation devised for dealing with heterogeneous problems. The problem was recasted in terms of interfaces variables, from which different interface variational formulations were derived. The conditions under which it is possible to have existence and uniqueness results of such different formulations were established, and the corresponding results were proved. Within this context, the decomposition of the original heterogeneous problem into homogeneous decoupled subproblems could be straightforwardly introduced. Additionally, it was possible to study some relevant properties of the resulting interface problem also in the case of a system with an arbitrary number of components. Finally, two examples of application were presented in order to confirm the numerical results obtained and to show the effectiveness and motivate the use of such models in certain applications.

\section{References}

1. Babuška, I.: The finite element method with Lagrangian multipliers. Numer. Math. 20, 179-192 (1973)

2. Badia, S., Nobile, F., Vergara, C.: Robin-Robin preconditioned Krylov methods for fluid-structure interaction problems. Comput. Methods Appl. Mech. Eng. 198(33-36), 2768-2784 (2009)

3. Bernardi, C., Dauge, M., Maday, Y.: Spectral Methods for Axisymmetric Domains, volume Series in Applied Mathematics 3. Gauthier-Villars, Editions Scientifiques et Médicales Elsevier, Paris (1999)

4. Blanco, P.J., Feijoo, R.A.: Sensitivity analysis in kinematically incompatible models. Comput. Methods Appl. Mech. Eng. 198, 3287-3298 (2009)

5. Blanco, P.J., Feij6o, R.A., Urquiza, S.A.: A unified variational approach for coupling 3D-1D models and its blood flow applications. Comput. Methods Appl. Mech. Eng. 196, 4391-4410 (2007)

6. Blanco, P.J., Feijoo, R.A., Urquiza, S.A.: A variational approach for coupling kinematically incompatible structural models. Comput. Methods Appl. Mech. Eng. 197, 1577-1602 (2008)

7. Blanco, P.J., Pivello, M.R., Urquiza, S.A., Feij6o, R.A.: On the potentialities of 3D-1D coupled models in hemodynamics simulations. J. Biomech. 42, 919-930 (2009) 
8. Blanco, P.J., Urquiza, S.A., Feijóo, R.A.: Assessing the influence of heart rate in local hemodynamics through coupled 3D-1D-0D models. Int. J. Numer. Methods Biomed. Eng. 26, 890-903 (2010)

9. Deparis, S., Discacciati, M., Fourestey, G., Quarteroni, A.: Fluid-structure algorithm based on SteklovPoincaré operators. Comput. Methods Appl. Mech. Eng. 195, 5797-5812 (2006)

10. Discacciati, M., Quarteroni, A.: Convergence analysis of a subdomain iterative method for the finite element approximation of the coupling of Stokes and Darcy equations. Comput. Vis. Sci. 6, 93-103 (2004)

11. Eringen, A.C., Şuhubi, E.S.: Elastodynamics Volume II: Linear Theory. Academic Press, New York (1975)

12. Formaggia, L., Gerbeau, J.F., Nobile, F., Quarteroni, A.: On the coupling of 3D and 1D NavierStokes equations for flow problems in compliant vessels. Comput. Methods Appl. Mech. Eng. 191, 561-582 (2001)

13. Gastaldi, F., Gastaldi, L., Quarteroni, A.: Adaptive domain decomposition methods for advection dominated equations. East-West J. Numer. Math. 4(3), 165-206 (1996)

14. Grinberg, L., Anor, T., Madsen, J.R., Yakhot, A., Karniadakis, G.E.: Large-scale simulation of the human arterial tree. Clinical and Experimental Pharmacology and Physiology 36, 194-205 (2009)

15. Leiva, J.S., Blanco, P.J., Buscaglia, G.C.: Iterative strong coupling of dimensionally-heterogeneous models. Internat. J. Numer. Methods Eng. 81, 1558-1580 (2010)

16. Migliavacca, F., Balossino, R., Pennati, G., Dubini, G., Hsia, T.-Y., de Leval, M.R., Bove, E.L.: Multiscale modelling in biofluidynamics: application to reconstructive paediatric cardiac surgery. J. Biomech. 39, 1010-1020 (2006)

17. Quarteroni, A., Valli, A.: Numerical Approximation of Partial Diffential Equations. Springer, Berlin (1994)

18. Quarteroni, A., Valli, A.: Domain Decomposition Methods for Partial Differential Equations. The Clarendon Press, Oxford University Press, New York (1999)

19. Quarteroni, A., Veneziani, A.: Analysis of a geometrical multiscale model based on the coupling of PDE's and ODE's for blood flow simulations. SIAM J. Multiscale Model. Simul. 1, 173-195 (2003)

20. Quarteroni, A., Veneziani, A., Zunino, P. : A domain decomposition method for advection-diffusion processes with application to blood solutes. SIAM J. Sci. Comput. 23(6), 1959-1980 (electronic) (2002)

21. Rozza, G., Huynh, D.B.P., Nguyen, N.C., Patera, A.T.: Real-time reliable simulation of heat transfer phenomena. In: ASME-American Society of Mechanical Engineers. Heat Transfer Summer Conference Proceedings, S. Francisco, CA, US, July 2009. ASME, New York, (2009) Paper HT2009-88212

22. Smith, B.F., Bjørstad, P.E., Gropp, W.D.: Domain Decomposition. Cambridge University Press, Cambridge (1996)

23. Toselli, A., Widlund, O.: Domain Decomposition Methods-Algorithms and Theory, volume 34 of Springer Series in Computational Mathematics. Springer, Berlin (2005)

24. Urquiza, S.A., Blanco, P.J., Vénere, M.J., Feijóo, R.A.: Multidimensional modelling for the carotid artery blood flow. Comput. Methods Appl. Mech. Eng. 195, 4002-4017 (2006) 